\title{
Integrated Tool Set for Control, Calibration, and Characterization of Quantum Devices Applied to Superconducting Qubits
}

\author{
Nicolas Wittler®,${ }^{1,2,{ }^{*}, \dagger}$ Federico Roy $\odot,{ }^{1,3, \dagger}$ Kevin Pack, ${ }^{1,2}$ Max Werninghaus, ${ }^{1,3}$ Anurag Saha Roy $\odot,{ }^{1}$ \\ Daniel J. Egger $\odot,{ }^{3}$ Stefan Filipp, ${ }^{3,4,5}$ Frank K. Wilhelm, ${ }^{1,2}$ and Shai Machnes ${ }^{1,2}$ \\ ${ }^{1}$ Theoretical Physics, Saarland University, Saarbrücken 66123, Germany \\ ${ }^{2}$ Peter Grünberg Institut - Quantum Computing Analytics (PGI 12), Forschungszentrum Jülich, Jülich D-52425, \\ Germany \\ ${ }^{3}$ IBM Quantum, IBM Research GmbH, Zurich Research Laboratory, Säumerstrasse 4, Rüschlikon 8803, \\ Switzerland \\ ${ }^{4}$ Department of Physics, Technical University of Munich, Garching 85748, Germany \\ ${ }^{5}$ Walther-Meißner-Institut, Bayerische Akademie der Wissenschaften, Garching 85748, Germany
}

(Received 8 October 2020; revised 1 December 2020; accepted 10 February 2021; published 29 March 2021)

\begin{abstract}
Efforts to scale-up quantum computation have reached a point where the principal limiting factor is not the number of qubits, but the entangling gate infidelity. However, the highly detailed system characterization required to understand the underlying error sources is an arduous process and impractical with increasing chip size. Open-loop optimal control techniques allow for the improvement of gates but are limited by the models they are based on. To rectify the situation, we provide an integrated opensource tool set for control, calibration, and characterization $\left(\mathbb{C}^{3}\right)$, capable of open-loop pulse optimization, model-free calibration, model fitting, and refinement. We present a methodology to combine these tools to find a quantitatively accurate system model, high-fidelity gates, and an approximate error budget, all based on a high-performance, feature-rich simulator. We illustrate our methods using simulated fixedfrequency superconducting qubits for which we learn model parameters with less than $1 \%$ error and derive a coherence-limited cross-resonance gate that achieves $99.6 \%$ fidelity without the need for calibration.
\end{abstract}

DOI: 10.1103/PhysRevApplied.15.034080

\section{THE PROBLEM}

Scaling up quantum processing units (QPUs) is a monumental task that requires the community to make progress on multiple fronts, most importantly improving gate fidelities and increasing the number of qubits. Over the past few years, significant emphasis has been placed on creating larger devices, yielding great success [1,2]. However, the number of qubits has outstripped the limits that fidelity places on their utility. In Ref. [1], a record quantum volume [3] of 64 was demonstrated, loosely translating to the device being able to perform $\log _{2}(64)^{2}=36$ entangling gates before fidelity drops below $\frac{2}{3}$, a relatively small number of gates for an array of six qubits. In Ref. [2] the circuit fidelity was $0.1 \%$, thus requiring 30 million repetitions to

*c3@q-optimize.org,n.wittler@pm.me

$\dagger$ These authors contributed equally to this work.

Published by the American Physical Society under the terms of the Creative Commons Attribution 4.0 International license. Further distribution of this work must maintain attribution to the author(s) and the published article's title, journal citation, and DOI. achieve the desired statistics. One could even argue that the two-qubit gate fidelities demonstrated in isolation in 2014 [4] (0.994) are comparable with those in 2019 [2] (0.9938), even though the latter are for simultaneous gates in a large two-dimensional (2D) qubit array.

The relatively slow progress in improving gate fidelities can be traced back to an incomplete understanding of the sources of error. Indeed, characterization and calibration of QPUs to the desired accuracy is impractical and cumbersome, and operating on devices of increasing qubit number requires entangling gates to be fine tuned for each individual pair to account for slightly varying properties. The resulting lack of detailed models makes it harder to identify where efforts must be focused to achieve higher fidelity gates $[5,6]$.

Given that "all models are wrong, but some are useful" [7], we describe a Good Model as follows.

A Good Model is one that predicts the behavior of the system for the operations we wish to perform, to accuracies we care about.

For a QPU, a Good Model has to have predictive power for the range of feasible gate-generating pulses and for long sequences of such gates, to a fidelity accuracy of 
the order of $10^{-5}$. To the authors' knowledge, no such Good Model for a superconducting QPU has ever been published.

Since models serve as the basis to derive high-fidelity gates in open-loop optimal control [8-15], any inaccuracies of the model will inevitably degrade the experimental accuracy of the resulting gates. This problem is only partially ameliorated by the first-order insensitivity of optimized pulses to model inaccuracies [16,17]. Methodologies such as the adaptive hybrid optimal control (Ad-HOC) protocol [18] — which combines a model-based open-loop optimization with a closed-loop experimental calibration $[12,19]$ - address this issue but leave one in an unsatisfactory position as the need for calibration proves the inadequacy of the model: the root causes of the remaining infidelities are unexplained.

Conversely, if a Good Model is known, gates generated by open-loop optimal control will, by definition, work on the experiment, not requiring further closedloop calibration. This enables the use of complex pulses that would otherwise require time-consuming calibration. Such a Good Model would also provide an error budget through a process of exploratory interrogation - evaluating the potential performance of the system where certain limitations have been removed, i.e., asking "what if ...?" Therefore, extracting a Good Model efficiently and in a highly automated manner is key to improving fidelities and a crucial step of QPU scale up.

In this work we present $\mathbb{C}^{3}$, our proposed approach to control, calibrate, and characterize QPUs. The paper is organized as follows. We present the conceptual steps of $\mathbb{C}^{3}$ in Sec. II and illustrate the methodology by example in Sec. III, showing how these steps are implemented. Section IV includes a detailed description of the modeling, optimization procedures, the data comparison function, and relevant prior work. We conclude in Sec. V with a discussion of the effort's current status and long-term directions.

\section{II. $\mathbb{C}^{3}$ - CONTROL, CALIBRATION, AND CHARACTERIZATION}

Current methodology relies on tailored routines to extract individual parameters of the system's model (characterization) or fine-tune specific parameters of pulses used (calibration) [20,21]. This approach becomes cumbersome and impractical as the number of model and pulse parameters increases. With $\mathbb{C}^{3}$ we propose a different paradigm: optimizing a figure of merit that is sensitive to the set of parameters we care about. This eliminates the need to design per-parameter measurements, and thus provides a more general approach. $\mathbb{C}^{3}$ at its core is composed of three separate optimizations, respectively implementing the tasks of control, calibration, and characterization.
$C_{1}$. Given a model, find the pulse shapes that maximize fidelity with a target operation. Pulse shapes may be constrained by an ansatz or allow direct arbitrary waveform generator (AWG) parameterization.

$C_{2}$. Given pulse shapes, calibrate their parameters, if possible simultaneously, to maximize a figure of merit measured by the actual experiment, thus improving beyond the limits of a deficient model.

$C_{3}$. Given control pulses and their experimental measurement outcome, optimize model parameters to best reproduce the results. Enhance the model if needed.

The tasks of open-loop optimal control, $C_{1}$, and calibration, $C_{2}$, are fairly established in the community $[8-15,18$, 19]. To characterize the system and provide us with a Good Model, we introduce $C_{3}$, a tool to optimize model parameters by comparing model prediction to experimental data. We refer to this task as model learning. For this purpose, one requires an experimental data set containing information about the implemented pulses and the corresponding measurement outcomes. To test the model accuracy, we reproduce the data set, applying the same pulses to a simulation of the experiment, and compare the resulting outcomes: this provides a model match score to optimize. Initially, a candidate model is formulated based on previous information or intuition. If the model is suitable to explain the experiment, the optimization will converge to a near perfect match, thus providing numeric values for the model parameters. Instead, if the match is poor, the user supplies a new model that is either an extension or modification of the previous candidate, and the optimization is repeated. Depending on user choice, learned values are carried over to the parameters of the new model or discarded.

As heterogeneous experimental data is the foundation for model learning, we suggest using the three tasks of $\mathbb{C}^{3}$ in sequence, as shown in Fig. 1. However, their application is by no means limited to this use case and one may choose to view them as stand-alone routines. The same tools used to realize $C_{1}, C_{2}$, and $C_{3}$ can also be used to further interrogate the system to obtain a sensitivity analysis of the optimized model in light of the experimental data and a breakdown of possible error sources.

We note that the intertwining of control and characterization has been raised in the more general context of control theory [22-25]. In quantum technology, there are some works that combine two of the three tasks: AdHOC [18] calls for optimal control followed by calibration; a combination of model-based gradient calculations and experimental calibrations is proposed in Refs. [26,27], but the data gathered is not used to improve the system model; in Ref. [28] pulses are designed specifically for the purpose of reconstructing the noise spectrum. 


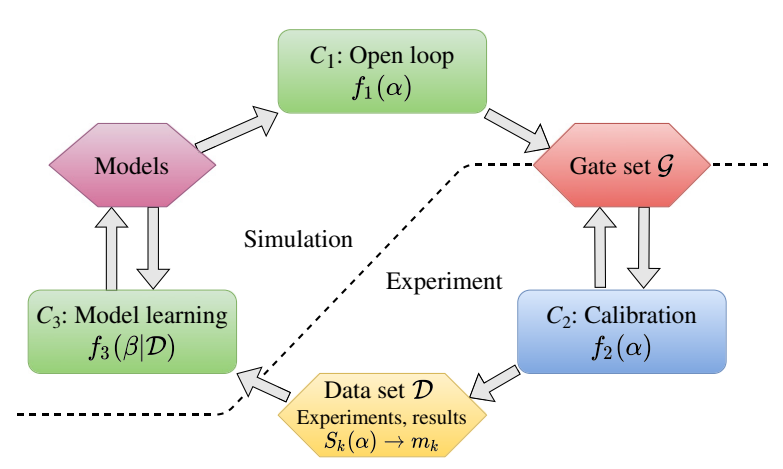

FIG. 1. Diagram of the $\mathbb{C}^{3}$ tool set in an integrated characterization loop. Here $C_{1}$ is a tool for obtaining optimal pulses by finding the control parameters $\alpha$ that minimize a goal function $f_{1}(\alpha)$ in simulation. The gate set $\mathcal{G}$ includes all the operations that one wishes to perform on the experiment, including the information of the ideal logical operations and the optimal pulse parameters $\alpha$ that implement them. By $C_{2}$ we denote a model-free experimental calibration procedure that optimizes pulse shapes with a gradient-free search to minimize an infidelity function $f_{2}(\alpha)$ by varying all parameters at once. A data set is a collection of experiment-result pairs, including information about the pulse parameters used, $\alpha$, the sequences $S_{k}$ performed, and the final outcomes measured, $m_{k}$. By $C_{3}$ we denote a tool for model learning that determines the model parameters $\beta$ that best explain the data set. It minimizes a goal function $f_{3}(\beta \mid \mathcal{D})$ obtained by recreating experiments $S_{k}(\alpha)$ in simulation and comparing the results to those in the experiment. In $\mathbb{C}^{3}$ different parameterized models can be provided to represent various elements of the experiment to find the one that best describes it. After the learning, the resulting model can be the basis for another characterization loop, refining both the model and controls.

\section{SYNTHETIC APPLICATION EXAMPLE}

The following synthetic example illustrates how $\mathbb{C}^{3}$ is used to obtain a Good Model in a realistic setting. We simulate a two-qubit QPU device using an underlying model, labeled the "real" model, which includes control discretization effects, electronics transfer functions, Markovian noise, and state preparation and measurement (SPAM) errors.

In this example, the simulated device is treated as a black box, which we interrogate with $\mathbb{C}^{3}$. We derive $\left(C_{1}\right)$ and calibrate $\left(C_{2}\right)$ optimal control pulses and use the resulting data to extract a Good Model $\left(C_{3}\right)$ by comparing the black box to three candidates.

Simple model. Two uncoupled qubits, closed system dynamics.

Intermediate model. Two coupled qubits, closed system dynamics.

Full model. Two coupled qubits, open system dynamics, including SPAM errors. Same structure as the "real" model, but a priori undetermined parameter values.

We systematically enrich the model until it reproduces the behavior of the device observed in $C_{2}$. The recovered model is then used to design a two-qubit gate that performs well on the black box without the need for tune up.

\section{A. The black-box device ("real" model)}

The "real" model is composed of two coupled threelevel Duffing oscillators, labeled $A$ and $B$, each directly driven by an external field $c_{i}(t)$. Initialization, dynamics, and readout are performed in the dressed basis by solving the master equation in Lindblad form [29,30],

$$
\dot{\rho}=-i[H, \rho]+\sum_{\substack{i=A, B, j=\phi, \kappa}} L_{i, j} \rho L_{i, j}^{\dagger}-\frac{1}{2}\left\{L_{i, j} L_{i, j}^{\dagger}, \rho\right\}
$$

with

$$
\begin{aligned}
\frac{H}{\hbar}= & \sum_{i=A, B}\left[\omega_{i} b_{i}^{\dagger} b_{i}-\frac{\delta_{i}}{2}\left(b_{i}^{\dagger} b_{i}-1\right) b_{i}^{\dagger} b_{i}\right] \\
& +g\left(b_{A}+b_{A}^{\dagger}\right)\left(b_{B}+b_{B}^{\dagger}\right)+\sum_{i=A, B} c_{i}(t)\left(b_{i}+b_{i}^{\dagger}\right),
\end{aligned}
$$

where $\omega_{i}$ is the frequency of qubit $i, \delta_{i}$ is the anharmonicity, $b_{i}\left(b_{i}^{\dagger}\right)$ is the lowering (raising) operator, and $g$ is the coupling strength. Open-system effects are expressed by the dephasing and relaxation Lindblad operators $L_{i, \phi}=$ $\sqrt{\frac{2}{T_{i}^{2 *}}} b_{i}^{\dagger} b_{i}$ and $L_{i, \kappa}=\sqrt{\frac{1}{T_{i}^{1}}} b_{i}$ with decay times $T_{i}^{2 *}$ and $T_{i}^{1}$.

Given the input drive signals $\varepsilon_{i}(t)$, we calculate the effective control fields $c_{i}(t)=\varphi\left[\varepsilon_{i}(t)\right]$, where the transfer function $\varphi$ [31] accounts for discretization introduced by the AWG, bandwidth limitations of hardware, and for a constant scaling $\varphi_{0}$, which translates input voltages to field amplitudes. We implement state preparation errors due to a nonzero initial temperature $T$ by starting each experiment from the thermal state

$$
\begin{gathered}
\rho_{\text {init }}=\frac{1}{Z}\left[|0\rangle\left\langle 0\left|+\exp \left\{-\frac{E_{1}}{k_{B} T}\right\}\right| 1\right\rangle\langle 1|\right. \\
\left.+\exp \left\{-\frac{E_{2}}{k_{B} T}\right\}|2\rangle\langle 2|\right],
\end{gathered}
$$

where $Z=\sum_{k=0}^{2} \exp \left\{-E_{k} / k_{B} T\right\}$ is the partition function with energies $E_{0,1,2}=0, \hbar \omega_{q}, \hbar\left(2 \omega_{q}+\delta\right)$, and $k_{B}$ is the Boltzmann constant. Readout misclassification is included, measuring state $|n\rangle$ as state $|m\rangle$ with probability $p_{n \rightarrow m}$. For example, the probability of measuring a state $\rho_{\psi}=|\psi\rangle\langle\psi|$ as $|0\rangle\langle 0|$ is

$$
\begin{aligned}
\Pi_{0}\left(\rho_{\psi}\right)= & p_{0 \rightarrow 0}\left\langle 0\left|\rho_{\psi}\right| 0\right\rangle+p_{1 \rightarrow 0}\left\langle 1\left|\rho_{\psi}\right| 1\right\rangle \\
& +p_{2 \rightarrow 0}\left\langle 2\left|\rho_{\psi}\right| 2\right\rangle .
\end{aligned}
$$

Similarly to the experiment, populations are estimated by averaging the results of multiple projective measurements, 
TABLE I. Overview of the parameters of the "real" model (reference values) and the candidate models, before and after the $C_{3}$ learning for different data sets. Candidate model values are shown as the difference from reference values. Dashes $(-)$ indicate parameters not present in the model, quotation marks (") indicate parameters not being changed.

\begin{tabular}{|c|c|c|c|c|c|c|c|c|c|c|c|}
\hline \multirow{2}{*}{$\begin{array}{l}\text { Parameter } \\
\text { Learning }\end{array}$} & \multirow{2}{*}{$\begin{array}{l}\text { Real } \\
\text { Model }\end{array}$} & \multicolumn{2}{|c|}{ Simple } & \multicolumn{2}{|c|}{ Intermediate } & \multicolumn{2}{|c|}{ Full (ORBIT) } & \multicolumn{2}{|c|}{$\mathrm{ORBIT}+\mathrm{QPT}$} & \multicolumn{2}{|c|}{ Decoherence } \\
\hline & & Before & After & Before & After & Before & After & Before & After & Before & After \\
\hline$\omega^{(A)}(\mathrm{MHz})$ & 5000 & -1.000 & -0.886 & -1.000 & -0.230 & -0.230 & +0.004 & +0.004 & -0.016 & $"$ & $"$ \\
\hline$\delta^{(A)}(\mathrm{MHz})$ & 210 & +1.000 & +0.702 & +1.000 & +0.281 & +1.000 & +0.400 & +0.4008 & +0.017 & $"$ & $"$ \\
\hline$\omega^{(B)}(\mathrm{MHz})$ & 5600 & +1.000 & +0.592 & +1.000 & +0.013 & +0.013 & -0.003 & -0.003 & +0.006 & $"$ & $"$ \\
\hline$\delta^{(B)}(\mathrm{MHz})$ & 240 & +1.000 & +0.981 & +1.000 & +4.32 & +1.000 & -0.016 & -0.016 & -0.005 & ” & $"$ \\
\hline$\varphi_{0}(\mathrm{MHz} / \mathrm{V})$ & 159.2 & +1.592 & -1.634 & +1.592 & -0.802 & -0.802 & +0.123 & +0.123 & +0.246 & $"$ & $"$ \\
\hline$g(\mathrm{MHz})$ & 20 & - & - & +1.000 & -0.665 & -0.665 & +0.046 & +0.046 & -0.119 & $"$ & $”$ \\
\hline$T(\mathrm{mK})$ & 50 & - & - & - & - & +5.000 & -3.172 & -3.172 & -0.216 & $"$ & $"$ \\
\hline$T_{1}^{(A)}(\mu \mathrm{s})$ & 27 & - & - & - & - & +4.000 & +4.439 & +4.439 & +0.021 & +0.021 & +0.738 \\
\hline$T_{2 *}^{(A)}(\mu \mathrm{s})$ & 39 & - & - & - & - & +2.000 & +1.994 & +1.994 & -2.353 & -2.353 & -0.020 \\
\hline$T_{1}^{(B)}(\mu \mathrm{s})$ & 23 & - & - & - & - & +3.000 & +4.543 & +4.543 & +5.704 & +5.704 & +0.666 \\
\hline$T_{2 *}^{(B)}(\mu \mathrm{s})$ & 31 & - & - & - & - & +5.000 & +6.183 & +6.183 & +4.716 & +4.716 & +0.897 \\
\hline$p_{0 \rightarrow 0}^{(A)}(\%)$ & 97 & - & - & - & - & -2.00 & -0.84 & -0.84 & -0.11 & ” & $"$ \\
\hline$p_{1 \rightarrow 1}^{(A)}(\%)$ & 96 & - & - & - & - & +0.20 & +0.38 & +0.38 & +0.02 & ” & ” \\
\hline$p_{0 \rightarrow 0}^{(B)}(\%)$ & 96 & - & - & - & - & -2.00 & -0.62 & -0.62 & -0.03 & 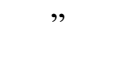 & ” \\
\hline$p_{1 \rightarrow 1}^{(B)}(\%)$ & 95 & - & - & - & - & +0.20 & +0.08 & +0.08 & +0.01 & " & ” \\
\hline
\end{tabular}

simulated as a multinomial draw from the distribution with probabilities $\left\{\Pi_{n}\right\}$, thus introducing noise stemming from a finite number of measurement repetitions (commonly known as "shot noise"). The values of the "real" model parameters are summarized in Table I.

\section{B. Open-loop optimal control, $C_{1}$}

We assume that at the start of the $\mathbb{C}^{3}$ procedure the parameters of the system are only known to a rough precision, with its qubit frequencies and anharmonicities chosen to be within a few megahertz of their "true" values. In the simple model, the qubits are uncoupled three-level Duffing oscillators, evolution follows closed system dynamics, and state preparation and measurement are assumed perfect. The Hamiltonian is

$$
\begin{aligned}
H / \hbar= & \sum_{i=A, B} \omega_{i} b_{i}^{\dagger} b_{i}-\frac{\delta_{i}}{2}\left(b_{i}^{\dagger} b_{i}-1\right) b_{i}^{\dagger} b_{i} \\
& +c_{i}(t)\left(b_{i}+b_{i}^{\dagger}\right) .
\end{aligned}
$$

Assuming this model, we design pulses for single-qubit gates using $C_{1}$. To mitigate leakage, we choose a pulse ansatz with a Gaussian shape and a correction given by the derivative removal by adiabatic gate (DRAG) method [14],

$$
\begin{aligned}
\varepsilon(t)= & A \Omega_{\mathrm{Gauss}}(t) \cos \left[\left(\omega_{d}+\omega_{\mathrm{off}}\right) t+\phi_{x y}\right] \\
& -\frac{\eta}{\delta} A \dot{\Omega}_{\mathrm{Gauss}}(t) \sin \left[\left(\omega_{d}+\omega_{\mathrm{off}}\right) t+\phi_{x y}\right] .
\end{aligned}
$$

Here, $\Omega_{\text {Gauss }}$ is a Gaussian envelope, $\dot{\Omega}_{\text {Gauss }}(t)$ is its time derivative, $A$ is the amplitude of the drive, $\omega_{\text {off }}$ is a frequency offset, and the DRAG parameter $\eta$ can be adjusted to reduce leakage into the second excited state $[14,33]$. The rotation axis can be freely chosen in the $x-y$ plane by changing the phase of the drive signal $\omega_{d} t \rightarrow$ $\omega_{d} t+\phi_{x y}$, implementing the unitary rotations $R\left(\phi_{x y}, \theta\right)=$ $\exp \left\{-i\left(\cos \phi_{x y} \sigma_{x}+\sin \phi_{x y} \sigma_{y}\right) \theta\right\}$. By setting $\phi_{x y}=n(\pi / 2)$ with $n=0,1,2,3$ and changing $\alpha=\left(A, \eta, \omega_{\text {off }}\right)$ we aim to realize the single-qubit gate set

$$
\mathcal{G}=\left\{X_{\pi / 2}, Y_{\pi / 2}, X_{-\pi / 2}, Y_{-\pi / 2}\right\}
$$

for each qubit separately, eight gates in total, where $X_{\pi / 2}=$ $\{R(0, \pi / 2)\}$ and so on. With $C_{1}$ we use a gradient-descent method to find the parameters $\alpha$ that minimize the mean average gate infidelity

$$
f_{1}(\alpha)=1-\frac{1}{|\mathcal{G}|} \sum_{U \in \mathcal{G}} f_{\text {av }}(U)=1-\frac{1}{|\mathcal{G}|} \sum_{U \in \mathcal{G}} \frac{\chi_{0,0} d+1}{d+1},
$$

where, $\chi_{0,0}$ is the $(0,0)$ th element of the chi matrix representation of the gate error $U^{\dagger} \cdot \widetilde{U}(\alpha)$ between the ideal gate $U$ and the implemented gate $\widetilde{U}(\alpha)$ [34]. We optimize Gaussian pulses with a gate length of $t_{g}=7 \mathrm{~ns}$, for both qubits, using the gradient-based Limited-memory Broyden-Fletcher-Goldfarb-Shanno (L-BFGS) algorithm [36]. The obtained optimal pulses yield mean infidelities of $f_{1}(\alpha)=6.6 \times 10^{-4}$ and $f_{1}(\alpha)=4.9 \times 10^{-4}$ on the simple model for qubit $A$ and qubit $B$, respectively - realistic 
values for fast gates using this simple parametrization. Next, we compare the performance of these pulses on the black-box device, where the gates instead yield a mean infidelity of $2.4 \times 10^{-3}$ for qubit $A$ and $1.5 \times 10^{-3}$ for qubit $B$. In fact, performing an experimentally realistic randomized benchmarking (RB) [37-40] measurement on the device yields errors per gate of $2.3 \times 10^{-3}$ and $1.3 \times 10^{-3}$ comparable with the theoretical average infidelity. The degradation of performance from optimal control simulation (approximately $10^{-4}$ ) to experiment (approximately $10^{-3}$ ) shows a clear mismatch between the device and the simple model.

\section{Calibration, $\boldsymbol{C}_{2}$}

The next step is to calibrate the pulses derived by $C_{1}$ and improve their performance on the device. We use $C_{2}$ and employ a closed-loop, model-free, gradient-free optimization algorithm on an experimentally accessible figure of merit $f_{2}$. Since the goal is to evaluate a gate set, we choose $f_{2}$ to be the result of optimized randomized benchmarking for immediate tune up (ORBIT) [19]

$$
f_{2}(\alpha)=f_{\text {ORBIT }}(\alpha)=\frac{1}{N} \sum_{k=1}^{N}\left[1-m_{k}(\alpha)\right],
$$

averaging over $N$ sequences. The survival probability, $m_{k}=\Pi_{0}\left[S_{k}(\alpha) \rho_{\text {init }} S_{k}^{\dagger}(\alpha)\right]$, is the probability to measure the state $|0\rangle$ [see Eq. (4)] after applying random sequences

$$
S_{k}(\alpha):=\left\{\prod_{j}^{L-1} C_{k, j}\right\} C_{\mathrm{inv}}
$$

composed of $L$ Clifford gates [19], to the initial thermal state $\rho_{\text {init }}$. The $C_{k, j}$ are the random gates sampled from the Clifford group $C$ (for a single qubit $|C|=24$ ), and $C_{\text {inv }}$ is chosen so that $S_{k} \equiv \mathcal{I}$ in the ideal case. We use the atomic operations $\mathcal{G}$ from Eq. (7) to construct the set of Clifford gates, e.g., $C_{6}=X_{-\pi / 2} \circ Y_{-\pi / 2} \circ X_{\pi / 2}$, and from them construct $N=25 \mathrm{RB}$ sequences of length $L=100$. The survival probabilities $m_{k}$ are estimated by performing $s=1000$ projective measurements and averaging.

To minimize $f_{2}$, we employ the covariance matrix adaptation evolution strategy (CMA-ES) algorithm [41] a gradient-free search that samples the loss function in batches, and is fairly robust to local minima and noise [42]. See Ref. [32] for an experimental demonstration. The optimal pulse parameters from $C_{1}$ are used as the starting point of the optimization, and the parameterization is kept as in Eq. (6). We perform the calibration for each qubit independently, with similar results. See Fig. 2 for the ORBIT calibration data of qubit $B$. The initial point suggested by $C_{1}$ has an ORBIT infidelity of 0.50 and is improved by the optimization to 0.12 . To account for SPAM errors, we perform a full RB measurement and estimate the infidelity of

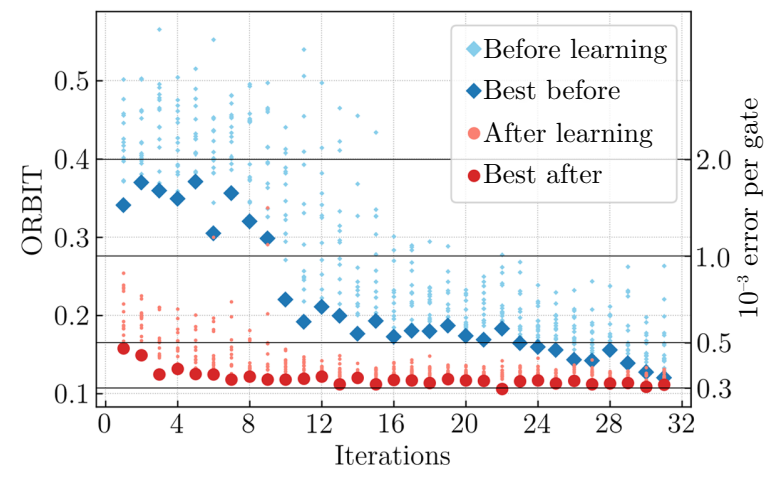

FIG. 2. The $C_{2}$ calibration on the device for single-qubit gates of qubit $B$. The initial point is suggested by $C_{1}$ before (after) learning of the model. The light blue diamonds (light red circles) represent the values of the ORBIT goal function, Eq. (9), for varying pulse parameters $\alpha$ as chosen by the search algorithm. The larger blue diamonds (larger red circles) highlight the best of 25 points generated and sampled at each iteration. In experimental practice, this batching helps reduce the overhead of loading pulses in AWG programming [32]. Both calibrations achieve the same final fidelity; however, the optimal gates derived from the learned model provide a better initial guess. Assuming no SPAM errors, the ORBIT value can be translated into an error per gate, indicated on the right axis. This is only meant to provide a rough estimate of the performance of the gate, noting that an ORBIT value of 0.5 represents maximum error per gate, i.e., completely depolarizing channels.

the gates before and after as $1.3 \times 10^{-3}$ and $3.4 \times 10^{-4}$, respectively. Qubit $A$ shows a similar improvement of RB estimated error from $2.3 \times 10^{-3}$ to $7.5 \times 10^{-4}$.

For the purpose of learning, we define the data set $\mathcal{D}:=\left\{S_{k}\left(\alpha_{j}\right) \rightarrow m_{j, k}\right\}$, the collection of the experiments conducted during the $C_{2}$ calibration, consisting of pulse parameters $\alpha_{j}$ and gate sequences $S_{k}\left(\alpha_{j}\right)$, and the corresponding measurement outcomes $m_{j, k}$.

\section{Characterization, $\boldsymbol{C}_{3}$}

In $C_{3}$, we use the data set $\mathcal{D}$ obtained during ORBIT calibration to improve the model of the system. For each measurement result $m_{j, k}$, we compute the equivalent simulation result $\widetilde{m}_{j, k}(\beta)$ by calculating the dynamics of the sequence $S_{k}\left(\alpha_{j}\right)$ given a set of model parameter values $\beta=\left(\omega_{i}, \delta_{i}, \ldots\right)$. Since simulating the whole data set is computationally costly, for the purpose of model learning, we make a selection of eight pulse parameter sets $j$ per qubit from the full data set. Each parameter set includes $k=1, \ldots, 25$ sequences, meaning that we learn from a total of $N=400$ measurement results, relabeled as $m_{n}$. We then construct a logarithmic likelihood (LL) goal function

$$
f_{3}(\beta)=f_{\mathrm{LL}}(\mathcal{D} \mid \beta)=\frac{1}{2 N} \sum_{n=1}^{N}\left[\left(\frac{m_{n}-\tilde{m}_{n}}{\widetilde{\sigma}_{n}}\right)^{2}-1\right]
$$


that captures how well the model prediction $\widetilde{m}_{n}$, with standard deviation $\widetilde{\sigma}_{n}$, agrees with the recorded values $m_{n}$. Because of the finite number of measurements, the averaged $m_{n}$ are noisy estimates of the population, with a mean $\mu_{n}$ and standard deviation $\sigma_{n}$. Thus, they cannot be matched perfectly even when all model parameters are exact. However, we can determine the expectation value of the goal function $f_{\mathrm{LL}}$ in the scenario where all $\widetilde{m}_{n}$ are exactly a given number of standard deviations away from the underlying true value $\mu_{n}$. A detailed mathematical discussion is presented in Sec. IV C. To provide a more intuitive measure, we express the match $f_{\mathrm{LL}}$ in terms of the number of standard deviations that would result in the same score.

To minimize $f_{3}(\beta)$, we use a combination of two algorithms: gradient free (CMA-ES algorithm) to avoid local minima and gradient based (L-BFGS algorithm) to converge quickly once the right minimum has been identified. In Fig. 3 we show the convergence of the $C_{3}$ optimization for different models. The simple model is not able to reproduce the device's results, as the optimization ends at approximately 8 standard deviations away. This demonstrates that the experiment on the device includes behavior not captured by the simple model. Spectator effects might be significant even when performing only single-qubit experiments, making the completely uncoupled model insufficient. Another source of this inconsistency might be

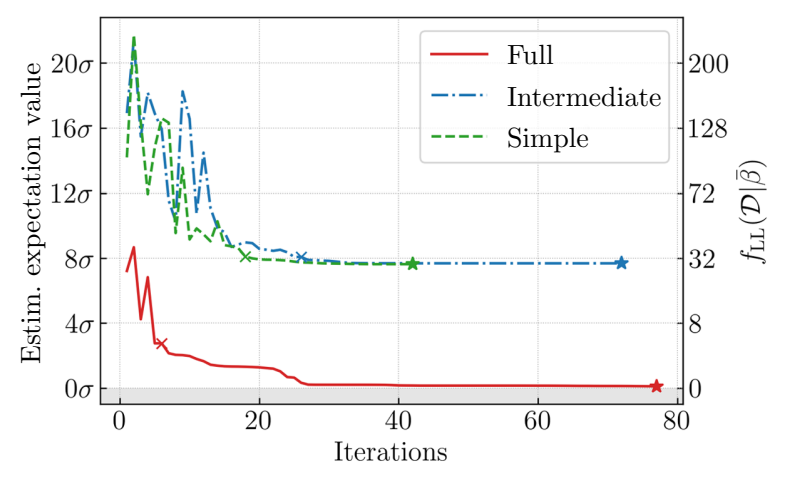

FIG. 3. Progress of the $C_{3}$ optimization on a hierarchy of models: simple model (green, dashed), intermediate model (blue, dot-dash), and full model (red, solid), as described in the text. The model match goal function $f_{\mathrm{LL}}(\beta)$ is defined in Eq. (11). The crosses show the switch over from the CMA-ES algorithm to the L-BFGS algorithm. The CMA-ES algorithm evaluates a batch of points for each iteration $(8,9$, and 12 for the simple, intermediate, and full model, respectively); only the best of each batch is shown. The L-BFGS algorithm takes on average approximately 1.2 evaluations per iteration, for all three models. The function $f_{\mathrm{LL}}$ is rescaled to express the match in terms of standard deviations of the binomial distribution that the experimental results are drawn from. The simple model is a close dispersive approximation of the intermediate model, demonstrated by their similar final match score. By including all relevant device properties, the full model reaches an almost perfect match score.
SPAM errors not accounted for in the model that might play a large role in actual measurement results. The parameter values resulting from this $C_{3}$ process and all following ones are shown in Table I.

Going forward an informed decision has to be made about how to enhance the model. Since the true values of the parameters are not known in an experimental setting, we require a tool to determine the precision to which they are learned. We estimate the sensitivity to changes of model parameters around the optimal values $\beta^{\prime}$ by performing one-dimensional scans and observing the degradation in the model match score, $f_{\mathrm{LL}}\left(\mathcal{D} \mid \beta^{\prime}+\delta \beta\right)$. In Fig. 4(a) we show that sweeping the value of frequency of qubit $B$ produces a highly irregular landscape of the match score $f_{\text {LL. }}$.

The simple model is then extended by adding the static coupling $g$ of unknown exact value, resulting in the intermediate model. When repeating $C_{3}$, we initialize model parameters from the initial, rough values. We do not carry over the learned parameters from the simple model to the intermediate model because, by introducing a coupling, we expect slightly shifted frequencies compared to the bare frequencies of the uncoupled qubits. Nonetheless, convergence of the match score shows no improvement from the simple model, still only reaching within approximately 8 standard deviations from experimental results (Fig. 3) and resulting in a similar sensitivity landscape in Fig. 4(a). This suggests that the simple model is a close dispersive approximation of the intermediate model. Indeed, we observe a dispersive shift [43] of $593 \mathrm{KHz}$, consistent with the expected $g^{2} /\left(\omega_{B}-\omega_{A}\right) \simeq 666 \mathrm{KHz}$, given the coupling of $g \simeq 20 \mathrm{MHz}$ and the frequency difference $\omega_{B}-\omega_{A} \simeq$ $600 \mathrm{MHz}$.

Finally, model complexity is increased by adding three relevant features: Markovian noise simulated by Lindblad master equation, initialization errors due to finite operating temperature, and measurement errors in the form of misclassification. The system model is now of the same structure as the "real" model of the device. Starting from the best intermediate model parameters, the $C_{3}$ procedure converges satisfactorily, approaching the 0 standard deviations mark (Fig. 3).

In Fig. 5(a) we show the value of each parameter of the full model during optimization, as we introduce different learning data (in the next sections), and compare with their true values (dashed lines). By learning the model parameters with the ORBIT data (white left section of each plot), the model frequencies $\omega_{A / B}$, anharmonicities $\delta_{A / B}$, coupling $g$, and line transfer function $\varphi_{0}$ converge to their true values. The temperature and misclassification parameters are not recovered, and we believe this is due to an extra degree of freedom that is not resolved by the experiments we have performed, as the effects of misclassification, Eq. (4), and initial thermal distribution, Eq. (3), are similar and can be partially exchanged. Dephasing 

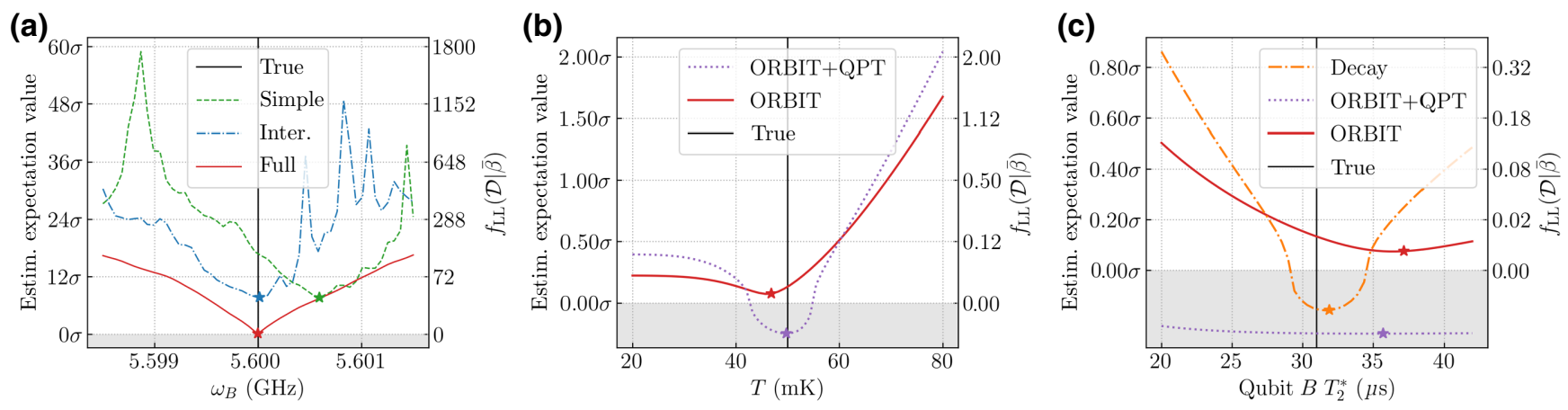

FIG. 4. Sensitivity analysis of selected parameters for different models and data sets. In (a) we sweep the qubit frequency $\omega_{B}$ and evaluate the goal function $f_{\mathrm{LL}}$ on the learning data at each value. The star represents the optimal value returned by $C_{3}$. Intermediate (blue, dot-dash) and full (red, solid) models show the same frequency value, while the value of the simple model (green, dashed) is dispersively shifted, as expected. In (b),(c) we perform the same sweep for the chip temperature and $T_{2}^{*}$ of qubit $B$, respectively, evaluated on the full model for different learning data sets. The ORBIT data are the same as used in the full model in (a). Introducing the quantum process tomography (QPT) data (purple, dotted) allows a more precise definition of the temperature. To determine $T_{2}^{*}$, we use relaxation and dephasing data (orange, dot-dash). Match values below 0 can occur because of noisy data, finite sampling, and deviation from the assumption of a Gaussian distribution of the data. More sensitivity plots are shown in the Supplemental Material [35].

and relaxation times (not shown) are also not recovered. Indeed, in Fig. 4(c) we show that the sensitivity of the data to dephasing time $T_{2}^{*}$ of qubit $B$ is minimal. RB sequences perform an effective random dynamical decoupling [44], providing a possible explanation to this result.

\section{E. Validation of the learned model}

After model matching on a subset of the data in the $C_{3}$ step, we now evaluate the predictive power of the learned models by computing the score on the rest of the data set (this is also known as a validation set in machine learning). This verifies that the selected subset captures all relevant behavior and alleviates the danger of overfitting.

Figures 6(a) to 6(c) depict the correlation between calibration data points $m_{j, k}$ and their model-based reconstructions $\tilde{m}_{j, k}$. We evaluate the goal function $f_{\mathrm{LL}}(\beta)$ over the validation set for the simple, intermediate, and full models and obtain values of 36.5 (approximately 8.4 $\sigma$ ), 42.0 (approximately 9.2 $\sigma$ ), and 0.028 (approximately $0.2 \sigma$ ), respectively. The conclusion is that, even though some parameters are not recovered by $C_{3}$, the learned full model is indeed a Good Model, as it reproduces the behavior of the system on all previously recorded data points to satisfying accuracy. This does not prevent additional measurement data to expose new behavior in the system: the notion of the Good Model is always tied to the underlying data set.

Furthermore, we now repeat the $C_{1}$ procedure on the Good Model (yielding average gate infidelities of $6.3 \times$ $10^{-4}$ and $1.1 \times 10^{-3}$ for qubits $A$ and $B$, respectively) and show that the resulting pulses give a near optimal performance on the actual device and allow for faster $C_{2}$ convergence, as seen in Fig. 2. One would expect the pulses derived from the Good Model to be exactly optimal on the actual device. Even though it is not the case here, this is not because of an inaccurate model, but rather because of a disparity between the figures of merit used in $C_{1}$ (average infidelity) and $C_{2}$ (single-qubit ORBIT). Average fidelity captures effects of the whole system, including, in this case, an effective $\mathrm{ZZ}$ coupling between the two qubits caused by a slight repulsion of the $|02\rangle$ and $|11\rangle$ states that are $300 \mathrm{MHz}$ apart. Minimizing a single-qubit ORBIT infidelity does not adjust for this effect, as we can verify by evaluating both $\mathrm{RB}$ (which captures only one qubit at a time) and average infidelity before and after calibration. Indeed, the average infidelity of qubit $B$ is $1.2 \times$ $10^{-3}$ (compatible with the performance of $1.1 \times 10^{-3}$ on the Good Model), but the error per gate is estimated by $\mathrm{RB}$ as $4.1 \times 10^{-4}$. After the calibration the RB estimate is improved to $2.9 \times 10^{-4}$, but the average infidelity is worsened to $1.9 \times 10^{-3}$. Performing simultaneous RB could resolve this issue.

\section{F. Entangling gate}

We further investigate the Good Model that was determined using only single-qubit calibration data by deriving a two-qubit cross-resonance (CR) gate $[45,46]$ with $C_{1}$. Both qubits are driven simultaneously at $\omega_{B}$, the resonant frequency of qubit $B$, to accumulate a phase $\pm \pi / 2$ conditioned on the state of qubit $A$ [20]. Both drives are parameterized by flattop Gaussians. The resulting CR pulse has a gate infidelity of $f_{\text {av }}=3.8 \times 10^{-3}$. When evaluated on the "real" model, the gate has an infidelity of $f_{\mathrm{av}}=4.3 \times 10^{-3}$, again showing that the learned model predicts device behavior to high accuracy. Notably, the model learned using only single-qubit data is sufficient to 

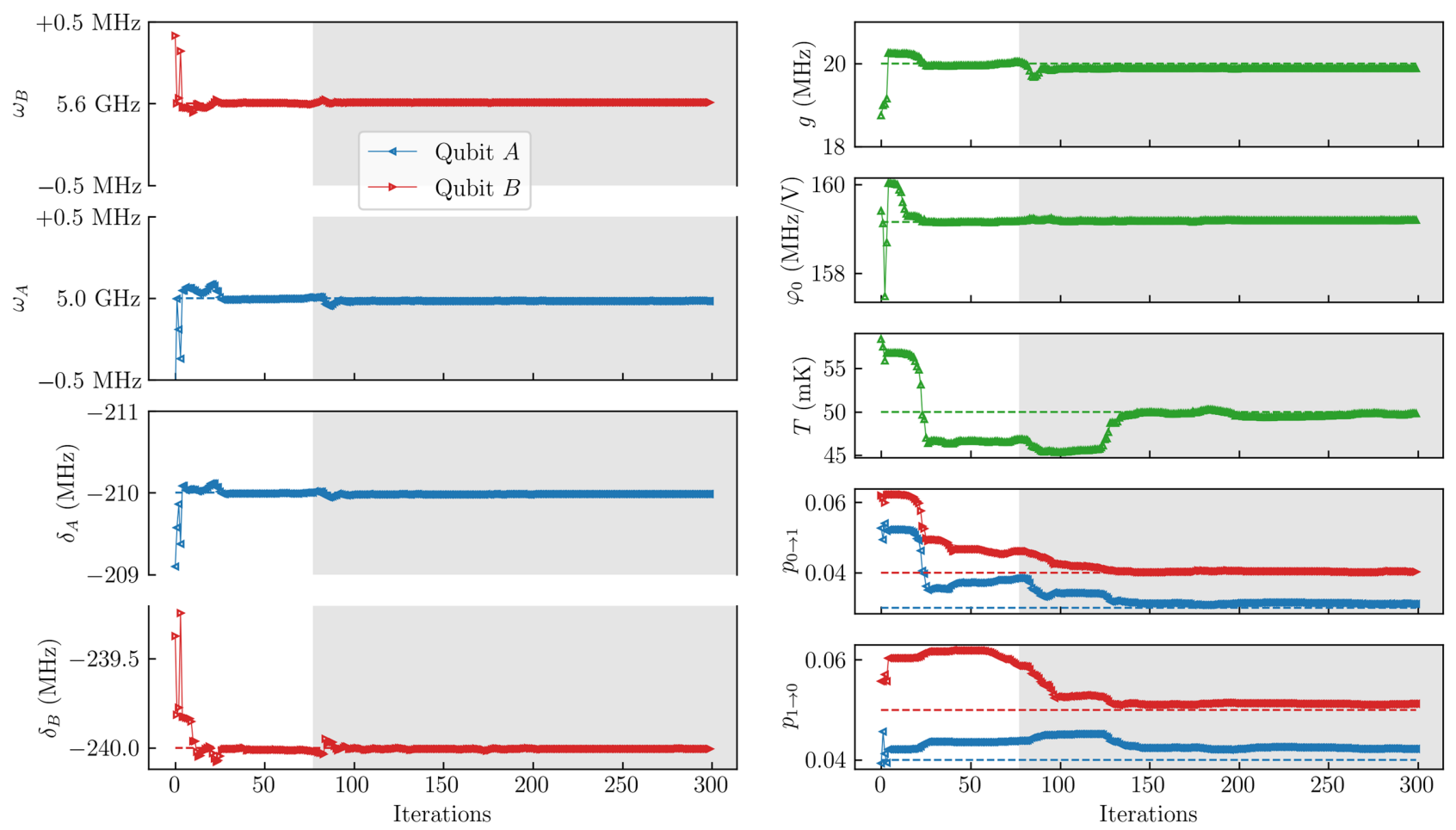

FIG. 5. The $C_{3}$ learning of the two-qubit model parameters. Blue, left (red, right) triangles indicate qubit $A(B)$ parameters, while shared properties are shown with green upward triangles. The true values of the "real" model are indicated as dashed lines. Learning begins using just ORBIT data (left white section) that fixes qubit frequencies, anharmonicities, coupling, and the line transfer function to their true values. Then tomography data from a two-qubit experiment are added (right gray section), which allows better identification of the chip temperature $T$ and the misclassification constants $p_{0 \rightarrow 1}$ and $p_{1 \rightarrow 0}$.

accurately predict the performance of the two-qubit gate on the device. We suspect this to be caused by exchange interactions due to coupling and finite temperature: Even when performing only single-qubit gates, the finite temperature causes a partial excitation of higher states, which are then exchanged with the other qubit via the coupling and thus visible in the ORBIT data.

The performance of the gate on the device is verified with QPT: we apply the CR gate preceded and followed by single-qubit gates to prepare and measure in the basis (a)

(b)

(c)

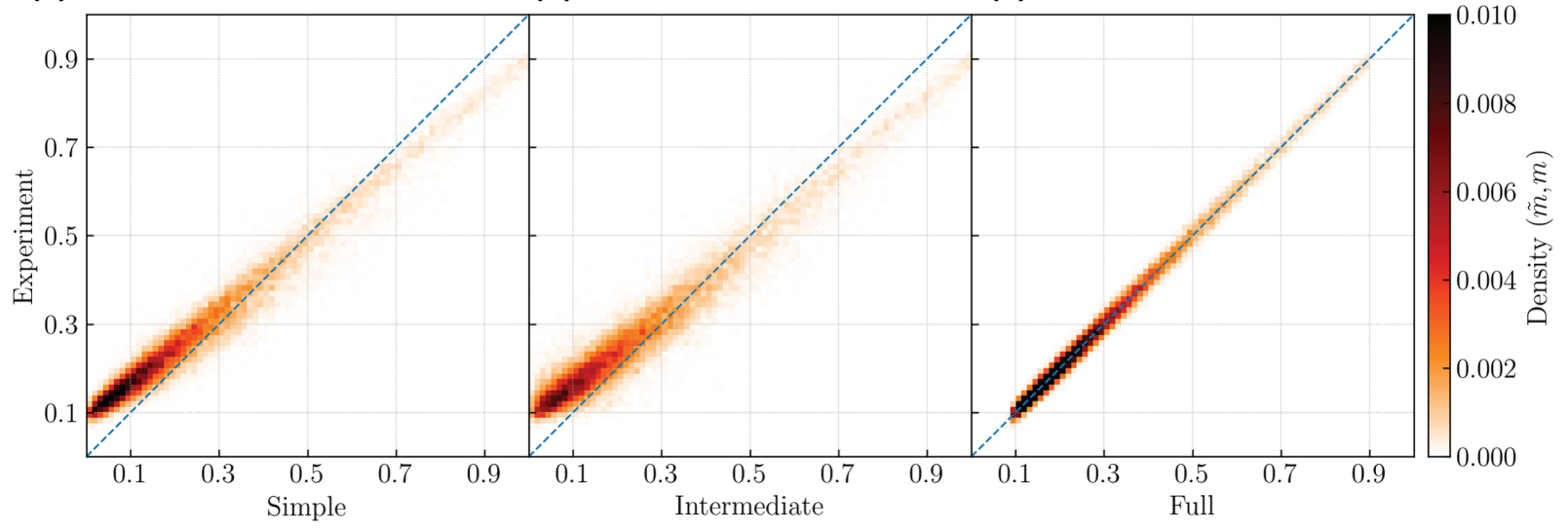

FIG. 6. (a)-(c) Correlation between simulation and experiment expressed as a density of points $\left(\tilde{m}_{k}, m_{k}\right)$ for the simple, intermediate, and full models, respectively. The data set is the so-called validation set: the data points $k$ that are not used in optimization of the model parameters. The simple and intermediate models show poor correlation as the simulation predicts a wide distribution of measurement outcomes for each recorded value. They also exhibit a tilt that can be attributed to SPAM errors not considered in the models. Only the full model produces a consistently high density distribution centered around the diagonal, with minimal spread due to the noisy data. 
states, e.g., $S=\left(X_{\pi / 2} \otimes Y_{\pi / 2}\right) \circ \mathrm{CR} \circ\left(X_{-\pi / 2} \otimes Y_{\pi / 2}\right)$ [47], and again collect these measurements into our data set. We believe that the entangling gate lifts the degree of freedom between misclassification and initial thermal distribution discussed before; hence, we now perform another $C_{3}$ optimization, using the QPT data (256 sequences) and one ORBIT parameter per qubit $(2 \times 25$ sequences $)$ as the learning data. Parameter convergence is shown in the gray areas of Fig. 5(a), where temperature and confusion matrix values are adjusted closer to the true values.

Figure 4(b) substantiates the claim that the entangling gate data allows for a more precise learning of the chip temperature, exhibiting a narrower valley at the true value. However, we are still not able to learn the $T_{1}$ and $T_{2}^{*}$ parameters, since the sequences in QPT are too short to be sensitive.

\section{G. Relaxation and dephasing}

To demonstrate how a specialized measurement is formulated within $\mathbb{C}^{3}$, we determine the values of $T_{1}$ and $T_{2}^{*}$, using simple established sequences that are known to be sensitive to these parameters. The decay lifetime $T_{1}$ is determined by preparing the excited state of the qubit, followed by increasing wait times, and then measuring the ground-state population. We write the sequence as

$$
S_{T 1}^{(n)}=X_{\pi / 2} \circ X_{\pi / 2} \circ \mathcal{I}^{n},
$$

where $X_{\pi / 2}$ is our previously optimized $\pi / 2$ gate and $\mathcal{I}^{n}$ signifies $n$ repetitions of the identity gate $\mathcal{I}$. Similarly,

$$
S_{T 2 *}^{(n)}=X_{\pi / 2} \circ \mathcal{I}^{n / 2} \circ X_{\pi / 2} \circ X_{\pi / 2} \circ \mathcal{I}^{n / 2} \circ X_{-\pi / 2}
$$

defines a Ramsey echo sequence, used to measure the dephasing time $T_{2}^{*}$. We take 51 logarithmically spaced values of $n$ between 100 and 10000 to capture the full decay curves.

Using this data set, we perform another $C_{3}$ optimization, freezing all model parameters learned until now and varying only the values of $T_{1}$ and $T_{2}^{*}$. By doing so we manage to determine their values to within $1 \mu \mathrm{s}$ of the true values (Fig. 7). This procedure is the $\mathbb{C}^{3}$ equivalent of a common exponential decay fit to the data. However, with $\mathbb{C}^{3}$, one does not require prior knowledge on the expected structure of the experimental results, i.e., an exponential decay. Hence, when matching the data, $\mathbb{C}^{3}$ also accounts for SPAM errors without the need to adjust the fitting function.

In Fig. 4(c) we show the sensitivity of $f_{\mathrm{LL}}$ to the value of $T_{2}^{*}$ of qubit $B$. The new data shows a clear improvement in the accuracy of the value obtained and the minimum is better defined. For increased sensitivity, one would require more decay data to learn from.
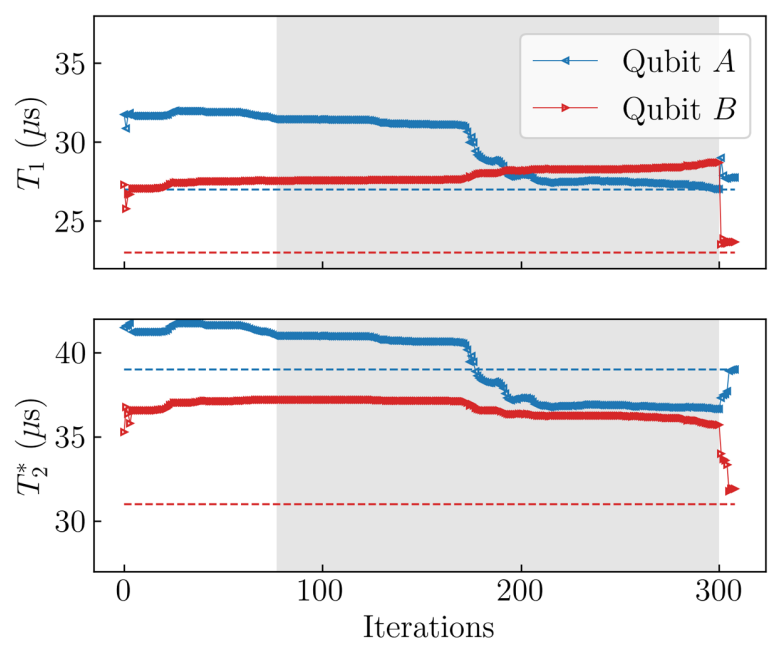

FIG. 7. The $C_{3}$ learning of the relaxation $\left(T_{1}\right)$ and dephasing $\left(T_{2}^{*}\right)$ parameters. Blue, left (red, right) triangles indicate qubit $A$ $(B)$ parameters. The true values of the "real" model are indicated as dashed lines. Background sections represent different learning data sets: just ORBIT data (left white section), a mix of ORBIT and QPT data (center gray section), decay and Ramsey data (right white section). The decay times are correctly identified only when specific data, sensitive to the decoherence effects, are used for learning, at which point they quickly converge to the real values.

\section{H. Sources of error}

The Good Model allows us to break down which of the model properties are preventing higher gate fidelities. To this end, we investigate the Good Model for components limiting the performance of the CR gate by idealizing aspects of the model.

We investigate whether the Gaussian ansatz is limiting gate fidelities by further refining the optimal pulses using a piecewise constant (PWC) optimization with one pixel per AWG sample (as is done in Ref. [32]). Average infidelity improves only marginally from $f_{\mathrm{av}}^{\mathrm{DRAG}}=3.8 \times 10^{-3}$ to $f_{\mathrm{av}}^{\mathrm{PWC}}=3.6 \times 10^{-3}$, suggesting other factors are limiting fidelities.

To find out if performance is limited by decoherence effects, we reoptimize the CR gate while disabling Lindbladian dynamics. By open-loop optimization in this idealized coherent setting, the error is decreased from $3.8 \times 10^{-3}$ to $1.3 \times 10^{-5}$. Thus, the $100 \mathrm{~ns} \mathrm{CR}$ gate considered here is coherence limited, as is the case in most experimental implementations [20,48], making improvements in gate time essential [49].

\section{IV. $\mathbb{C}^{3}$ IN DEPTH}

In the following we provide a detailed description of the $\mathbb{C}^{3}$ tool set, its modeling capabilities, and a general formulation of the optimization problems discussed in the previous section. 


\section{A. Experimental modeling}

To combine control and characterization, $\mathbb{C}^{3}$ provides a detailed simulation that endeavors to encompass all relevant practical considerations of the experiment such as signal processing, SPAM errors, control transfer functions, and Markovian noise. The simulator is used as the basis of the open-loop optimal control optimization $\left(C_{1}\right)$ and the model parameter optimization $\left(C_{3}\right)$. In both cases it is desirable to use gradient-based optimization algorithms $[10,50]$. However, it is extremely cumbersome to manually derive the full analytical gradients of the quantum dynamics, especially when it includes the properties described above. Instead, $\mathbb{C}^{3}$ uses a numerics framework [51] that allows for automatic differentiation [52], making the tool set more flexible and easily extendable. A similar approach is also used in Refs. [28,53] for control and characterization.

\section{Signal processing}

The simulation allows for the specification of control signals $\varepsilon(t)$ as either analytical functions or as direct, piecewise constant AWG parameterization. Analytic parameterizations are sampled at the resolution of the waveform generator producing the envelope signal $\varepsilon_{i}=\varepsilon\left(t_{i}\right)$, representing voltages being applied to the control line, where the $\left\{t_{i}\right\}$ are the AWG sample times. The resulting signal will exhibit a rise time $\tau$, due to the finite bandwidth of the control electronics. We model this by applying a convolution

$$
\widetilde{\varepsilon}(t)=\int_{t_{0}}^{t_{f}} \operatorname{interp}\left(\left\{\varepsilon_{i}\right\}\right)(t) G\left\{t_{f}-t\right\} d t
$$

with

$$
G(t)=\frac{1}{N} \exp \left\{-\frac{(t-\tau / 2)^{2}}{8 \tau^{2}}\right\},
$$

modeling a Gaussian filter, and

$$
\operatorname{interp}\left(\left\{\varepsilon_{i}\right\}\right)(t)=\left\{\varepsilon_{i} \mid t_{i} \leq t<t_{i+1}\right\}
$$

interpolating the sampled signal to higher resolution for simulation. An in-phase and quadrature mixer (IQ mixer) combines this envelope with a local oscillator signal of frequency $\omega_{\mathrm{lo}}$ to

$$
u(t)=I(t) \cos \left(\omega_{\mathrm{lo}} t\right)-Q(t) \sin \left(\omega_{\mathrm{lo}} t\right),
$$

where the in-phase and quadrature components

$$
\begin{aligned}
I(t) & =\widetilde{\varepsilon}(t) \cos \left(\phi_{x y}-\omega_{\text {off }} t\right), \\
Q(t) & =\widetilde{\varepsilon}(t) \sin \left(\phi_{x y}-\omega_{\text {off }} t\right)
\end{aligned}
$$

are assigned by a control parameter $\phi_{x y}$ and modulated to introduce a frequency offset $\omega_{\text {off }}$ on the drive. As noted in Ref. [54], in practice, there will be additional errors during the mixing, which are not currently modeled. In transmitting this signal to the experiment, various distortions can occur, modeled by a response function $\varphi$, which also converts the field from line voltage to an amplitude $c(t)=\varphi[u(t)]$.

\section{Time evolution}

The system Hamiltonian is

$$
H(t)=H_{0}+\sum_{k} c_{k}(t) H_{k}
$$

with a drift $H_{0}$ and optional control Hamiltonians $H_{k}$. The dynamics of the system are described by the time-ordered propagator

$$
U(t)=\mathcal{T} \exp \left\{-\frac{\mathrm{i}}{\hbar} \int_{t_{0}}^{t} H\left(t^{\prime}\right) d t^{\prime}\right\}
$$

given by solving the time-dependent Schrödinger equation, and approximated numerically by $U(t) \simeq \prod_{i=N}^{0} U_{i}$. Here, $U_{i}=\exp \left\{-(i / \hbar) H\left(t_{i}\right) \Delta t\right\}$, and the total time is divided into $N$ slices of length $\Delta t$ that are fine enough so that the Hamiltonian can be considered constant in the interval.

In application, we rarely perform a single gate or pulse in isolation. Experiments such as randomized benchmarking or the various flavors of tomography involve long pulse sequences that are inefficient to simulate as a whole. Instead, the $\mathbb{C}^{3}$ simulator computes each propagator $G$ of a defined gate set $\mathcal{G}$ individually and compiles these matrix representations into sequences. This avoids the need to solve the equations of motion multiple times for the same exact pulses. As the propagators are calculated in the dressed laboratory frame (as opposed to the single-particle rotating frame), consecutive gates need to be adjusted to realign with the rotating frame of the drive signal, by applying a $Z$ rotation with an angle of $\left(\omega_{\mathrm{lo}}+\omega_{\text {off }}\right) t_{g}$ [54].

To include open-system effects, we apply the equivalent procedure to obtain the process matrix

$$
\mathcal{E}(t)=\mathcal{T} \exp \left\{\int_{t_{0}}^{t} \mathcal{L}\left(t^{\prime}\right) d t^{\prime}\right\}
$$

by solving the master equation in Lindblad form,

$$
\dot{\rho}=\mathcal{L}(\rho)=-i[H, \rho]+\sum_{j} L_{j} \rho L_{j}^{\dagger}-\frac{1}{2}\left\{L_{j} L_{j}^{\dagger}, \rho\right\},
$$

where $H$ is the Hamiltonian from Eq. (19), the $L_{j}$ are Lindblad operators, and $\mathcal{L}$ is the generator in superoperator form [55]. The evolution of a state is obtained by applying the propagator as $\rho_{f}=U\left(t_{g}\right) \rho_{i} U^{\dagger}\left(t_{g}\right)$ for coherent evolution or $\rho_{f}=\mathcal{E}\left(t_{g}\right)\left[\rho_{i}\right]$ for incoherent evolution. 


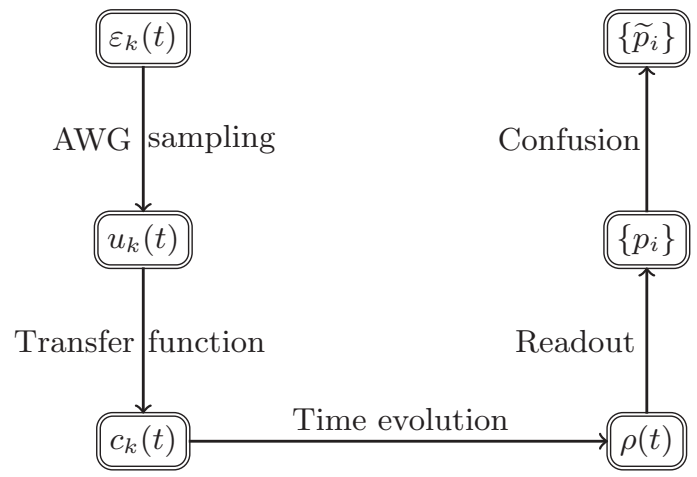

FIG. 8. The process of simulating experimental procedure for signal processing and readout. The $k$ th control function is specified by some function $\varepsilon_{k}(t)$ and specifies the line voltage $u_{k}(t)$ by an AWG with limited bandwidth. Electrical properties of the setup, such as impedances, are expressed as a line transfer function $\varphi$, resulting in a control field $c_{k}(t)=\varphi\left[u_{k}(t)\right]$, as in Eq. (19). After solving the equation of motion for the system, readout and misclassification are modeled by applying rescaling and transformations to the simulated populations $p_{i}=\left|\rho_{i i}\right|^{2}$, according to Eq. (24).

\section{Initialization and readout}

Given the temperature $T$ of the device, the system is initialized in a mixed state

$$
\rho_{\text {init }}=\sum_{k} \frac{1}{Z}\left|\phi_{k}\right\rangle\left\langle\phi_{k}\right| \exp \left\{-\frac{E_{k}}{k_{B} T}\right\},
$$

where $\left\{\left|\phi_{k}\right\rangle\right\}$ is the eigenbasis of $H_{0}$ and the normalization is given by the canonical partition function $Z=$ $\sum_{k} \exp \left\{-E_{k} / k_{B} T\right\}$

We simulate readout by postprocessing the final state $\rho_{f}$ : from the density matrix, represented in the dressed basis, we obtain a vector of populations $\vec{p}=\left(p_{k}\right)$ by taking the absolute square of the diagonal. This is consistent with a slow (or weak) readout scheme in experiment. Measurement and classification errors are modeled with a misclassification (confusion) matrix $\left(p_{i \rightarrow j}\right)_{i j}[56]$ such that the measured populations are

$$
\tilde{p}_{j}=\sum_{i} p_{i \rightarrow j} p_{i}
$$

To simulate an experimental measurement with an average of $l$ repetitions, we draw from a multinomial distribution of $l$ trails and with probabilities $\tilde{p}_{j}$.

\section{B. Optimizations}

For open and closed-loop optimal control as well as model learning, performing optimization processes is required.

\section{Open-loop model-based control: $\mathbf{C}_{1}$}

In the typical setting of open-loop optimal control $[8,9]$, given a model of a system, we search for the optimal control pulses to drive the system to a desired state or generate a certain gate. Pulses are parameterized by an analytic ansatz (e.g., Gaussian pulse with DRAG correction [14] to remove Fourier components coupling to leakage levels), or by direct AWG samples. Constraints may be imposed to conform with experimental feasibility, such as power and bandwidth limitations. The goal function to be minimized is selected depending on the specified optimal control task, e.g., state infidelity for state transfer problems, or unitary trace infidelity for quantum gates [9,34]. We suggest the use of average gate infidelity as the goal function, as it is experimentally accessible by performing RB or QPT, allowing comparison of performance in simulation and experiment.

Formally, the controls are parameterized as a vector of real numbers $\alpha$. Given a goal function $f_{1}(\alpha)$, we search for $\min _{\alpha} f_{1}(\alpha)$. Optimal control methods such as GRAPE [11], Krotov [13,57-59], and GOAT [10] have been devised to determine the gradient $\partial_{\alpha} f_{1}(\alpha)$ in order to facilitate convergence. These methods require a specific formulation of the problem and the analytical calculation of the gradient for any additional elements in the model, whereas in $\mathbb{C}^{3}$, automatic differentiation allows to systematically account for any model feature, including, for example, line response functions or SPAM error.

The disadvantage of gradient-based algorithms is their propensity to get trapped in local minima. The severity of the problem is reduced by using a hierarchy of progressively more complex control ansätze. If this is insufficient, a short preliminary gradient-free search to find the convergence basin most often resolves the problem.

\section{Closed-loop model-free calibration: $\mathrm{C}_{2}$}

In calibration, a given pulse is optimized to improve a figure of merit $f_{2}(\alpha)$, computed from experimental measurement results. In addition to gradient-free optimization algorithms, there are methods to approximate the gradients (e.g., Ref. [60]); however, such approaches are generally less efficient than gradient-free algorithms $[10,61]$ as they require a high number of evaluations [62]. If the initial point of the optimization is given by $C_{1}$, this implements the already established Ad-HOC [18] method. During calibration, sets of control parameters $\alpha_{j}$ are sent to the experimental setup, alongside instructions of how to evaluate the current controls. For evaluating gate sets, we suggest the ORBIT figure of merit, as it naturally performs a twirling of all sources of error, providing a single number to optimize. However, protocols tailored to specific needs can also be used, e.g., to obtain a desired conditional phase [5]. Then $C_{2}$ optimizes the control parameters $\alpha_{j}$ to minimize a figure of merit. 
While specialized measurements provide a straightforward way to fine tune controls related to specific device properties, they do not generally account for interdependency. For more complex setups with many parameters, such calibrations cannot be done without extraordinary effort [63]. In contrast, $\mathbb{C}^{3}$ employs modern gradient-free optimization algorithms, such as the CMA-ES algorithm (see Appendix B for further discussion), capable of optimizing dozens of parameters simultaneously, automating the task.

\section{Model learning: $\mathrm{C}_{3}$}

Extracting the model from a data set $\mathcal{D}$ can be thought of formally as analogous to the $C_{1}$ optimization task, where one varies the model parameters instead of the control parameters. For each measurement outcome $m_{k}$ in the data set,

$$
\mathcal{D}=\left\{S_{k} \mapsto m_{k}\right\}_{j},
$$

the corresponding gate or pulse sequences $S_{k}\left(\alpha_{j}\right)$ with control parameters $\alpha_{j}$ are used to simulate the model's prediction $\widetilde{m}_{j, k}=\tilde{m}\left[S_{k}\left(\alpha_{j}\right), \beta\right]$. The model learning goal function

$$
f_{3}(\mathcal{D} \mid \beta)=f_{3}\left(\left\{\tilde{m}_{k}(\beta)\right\},\left\{m_{k}\right\}\right)
$$

quantifies the match between the data set and the simulation of a system with parameters $\beta$. In this paper, we use a rescaled log likelihood

$$
f_{\mathrm{LL}}(\mathcal{D} \mid \beta)=\frac{1}{2 K} \sum_{k=1}^{K}\left[\left(\frac{m_{k}-\tilde{m}_{k}}{\tilde{\sigma}_{k}}\right)^{2}-1\right],
$$

where $\widetilde{\sigma}_{k}$ is the standard deviation of a binomial distribution with mean $\tilde{m}_{k}$, resulting in a variation of the Mahalanobis distance [64]. This function is strictly correct under the Gaussian assumption and a two-level readout. See Sec. IV C for the extension for a multiple outcome readout. The measurement process on any physical device is noisy, i.e., each $m_{k}$ is an estimate of a true underlying $\mu_{k}$. Therefore, a realistic data set $\mathcal{D}$ cannot be matched exactly by a deterministic simulation. The function $f_{\mathrm{LL}}$ is designed such that, for $n$ data points, its expectation value is 0 when the model predicts the means $\mu_{k}$ correctly, and $\frac{1}{2} n^{2}$ if the distance is $\mu_{k}-\widetilde{m}_{k}=n \sigma_{k}$ for all $k$, according to Eq. (32). This provides a more intuitive measure of the model match than the abstract value of $f_{\mathrm{LL}}$, i.e., it allows one to make a statement like "the model differs from the experiment by approximately $n$ standard deviations."

Because of the complexity of the physical systems, a potentially high number of interdependent parameters, and complex features of the landscape, it is difficult for the optimization to converge to the global optimum. Therefore, we take the tried-and-tested experimental approach of starting with a simple model and iteratively refining it. We modify the model and repeat the $C_{3}$ fit, optionally retaining the optimized parameters that are shared by the previous and new models. Alternatively, we collect additional data and repeat the optimization on the same model. We emphasize that at each of these steps the physicists' insights are required to evaluate the optimization's results, extend or discard models, and decide whether collecting additional data is required. Furthermore, employing a gradient-based algorithm can, depending on the initial point, result in a local minimum. The optimizations presented here were successful when starting with a gradient-free CMA-ES search, known to be robust against local minima, switching over to the faster converging gradient-based L-BFGS method when a promising parameter region is identified. However, further research is required to find the best optimization strategy.

Outside the countless parameter-specific measurements, there are many approaches that aim to automate or generalize the task of characterization, such as Bayesian learning (with Hamiltonian description [65-74], and more general purpose [75-81]), system identification [82-87], compressed sensing $[72,88,89]$, neural networks [90-92], and others [93].

In contrast, we note that in $\mathbb{C}^{3}$ a model takes explicit values for all its parameters, and is not represented as a high-dimensional distribution over model parameter space. This choice is driven by classical computation-load considerations: because the $\mathbb{C}^{3}$ model is highly detailed, and, as consequence, associated simulations are nontrivial, we believe a full Bayesian approach to any of $\mathbb{C}^{3}$ optimizations is not computationally viable at this time.

\section{C. $\mathrm{C}_{3}$ model fitting goal function}

When performing a series of experiments, $k \in$ $[1, \ldots, K]$, on a quantum device, each experiment $k$ is repeated a number of times and the normalized occurrences of the measurement outcomes are stored in a result vector $m_{k}$. These are collected in $\left\{m_{k}\right\}_{k=1, \ldots, K}$, or $\left\{m_{k}\right\}$ for shorthand. Given a model and its parameters $\beta$, we aim to quantify how likely it is that it underlies the observed data with a function $f_{3}\left(\left\{m_{k}\right\} \mid \beta\right)$. Hence, we need to determine the distance between the experimental result, $m_{k}$, and the model prediction, $\widetilde{m}_{k}(\beta)$. We define $p_{k}\left(m_{k} \mid \beta\right)$ to be the model-predicted probability distribution function (PDF) for the result of experiment $k$. As the $m_{k}$ are sampled from the readout distribution, we do not expect $m_{k}=$ $\widetilde{m}_{k}(\beta)$. Rather, we aim to define the function $f$ such that its expectation value, $E\left[f\left(\left\{m_{k}\right\}, \beta\right)\right]$, is zero if the underlying distributions from which the $\left\{m_{k}\right\}$ are drawn are the same as the model-predicted PDFs. 


\section{The Gaussian assumption}

To simplify calculation of $E\left[f\left(\left\{m_{k}\right\}, \beta\right)\right]$, we can make some assumptions regarding the underlying distributions. The natural $p_{k}\left(m_{k} \mid \beta\right)$ PDF is multinomial, determined by the dimension of the qubit Hilbert space $d_{k}$ (or binomial if dealing with a single qubit with no leakage levels). Under the assumption that, for a large number of shots, all possible readouts values are likely to appear, then by the central limit theorem (De Moivre-Laplace theorem) we can approximate $p_{k}$ with a multivariate normal distribution. Although, the multinomial distribution has a nondiagonal covariance matrix, one can diagonalize the distribution and decompose it as a product of one-dimensional Gaussian distributions. Thus, we write the PDF as a sum of $\sum_{k}\left(d_{k}-1\right)$ such distributions, redefine $K$ to equal the previous $\sum_{k}\left(d_{k}-1\right)$ and the $\left\{m_{k}\right\}$ to be their means.

\section{The model match distribution}

We use $\left\{\tilde{\mu}_{k}(\beta)\right\}$ and $\left\{\widetilde{\sigma}_{k}(\beta)\right\}$ to denote the mean and standard deviation of the model-predicted PDFs (after Gaussian assumption and multinomial diagonalization), and $\left\{\mu_{k}\right\}$ and $\left\{\sigma_{k}\right\}$ to denote the commensurate experimental values. We note that $\left\{\mu_{k}\right\}$ and $\left\{\sigma_{k}\right\}$ are unknown and unmeasured, and $\left\{m_{k}\right\}$ only provides an estimate of the mean. The simulation values $\left\{\tilde{m}_{k}\right\}$ on the other hand are deterministic and thus represent an exact estimate of the mean; hence, $\left\{\widetilde{m}_{k} \equiv \tilde{\mu}_{k}\right\}$.

The model-predicted PDF is given by the product of normalized Gaussian distributions, and gives the likelihood of the $\left\{m_{k}\right\}$ given the model parameters $\beta$ as

$$
\begin{aligned}
p\left(\left\{m_{k}\right\} \mid \beta\right) & =\prod_{k} p\left(m_{k} \mid \beta\right), \quad \text { where } \\
p\left(m_{k} \mid \beta\right) & =\frac{1}{\sqrt{2 \pi} \widetilde{\sigma}_{k}} \exp \left\{-\frac{1}{2}\left(\frac{m_{k}-\tilde{\mu}_{k}}{\tilde{\sigma}_{k}}\right)^{2}\right\}
\end{aligned}
$$

are the individual Gaussian distributions. We then construct the goal function as the average log likelihood, rescaled to give the desired expectation value,

$$
\begin{aligned}
f_{\mathrm{LL}}\left(\left\{m_{k}\right\} \mid \beta\right) & =-\log \left[K \sqrt[K]{p\left(\left\{m_{k}\right\} \mid \beta\right) \prod_{k} \sqrt{2 \pi} \widetilde{\sigma}_{k} \sqrt{e}}\right] \\
& =\frac{1}{K} \sum_{k} \frac{1}{2}\left[\left(\frac{m_{k}-\tilde{\mu}_{k}}{\tilde{\sigma}_{k}}\right)^{2}-1\right] .
\end{aligned}
$$

Here the $\sqrt[K]{\cdot}$ gives the average of the log likehoods, the $\sqrt{2 \pi} \widetilde{\sigma}_{k}$ removes the normalization of the Gaussians, such that they take value 1 when $m_{k}-\tilde{\mu}_{k}=0$, and the log likelihood is zero, and the $\sqrt{e}$ removes the residual part of the expectation caused by the noise in the $\left\{m_{k}\right\}$. Then, in the general case, when the Gaussians determined by the model are not the same as the Gaussians in the experimental data:

$$
\begin{aligned}
& E\left[f_{\mathrm{LL}}\left(\left\{m_{k}\right\} \mid \beta\right)\right] \\
& \quad=\frac{1}{2 K} \sum_{k}\left[\left(\frac{\mu_{k}-\tilde{\mu}_{k}}{\widetilde{\sigma}_{k}}\right)^{2}+\left(\frac{\widetilde{\sigma}_{k}}{\sigma_{k}}\right)^{2}-1\right], \\
& \operatorname{Var}\left[f_{\mathrm{LL}}\left(\left\{m_{k}\right\} \mid \beta\right)\right] \\
& \quad=\frac{1}{K^{2}} \sum_{k}\left(\frac{\sigma_{k}}{\widetilde{\sigma}_{k}}\right)^{2}\left[\left(\frac{\mu_{k}-\tilde{\mu}_{k}}{\widetilde{\sigma}_{k}}\right)^{2}+\frac{1}{2}\left(\frac{\sigma_{k}}{\widetilde{\sigma}_{k}}\right)^{2}\right] .
\end{aligned}
$$

In the limit that both distributions have the same standard deviation $\sigma=\widetilde{\sigma}$,

$$
\begin{aligned}
E\left[f_{\mathrm{LL}}^{\sigma \leftarrow \widetilde{\sigma}}\left(\left\{m_{k}\right\} \mid \beta\right)\right] & =\frac{1}{2 K} \sum_{k}\left(\frac{\mu_{k}-\tilde{\mu}_{k}}{\widetilde{\sigma}_{k}}\right)^{2}, \\
\operatorname{Var}\left[f_{\mathrm{LL}}^{\sigma \leftarrow \widetilde{\sigma}}\left(\left\{m_{k}\right\} \mid \beta\right)\right] & =\frac{1}{2 K}+\frac{1}{K^{2}} \sum_{k}\left(\frac{\mu_{k}-\tilde{\mu}_{k}}{\widetilde{\sigma}_{k}}\right)^{2} .
\end{aligned}
$$

Equation (32) then represents the square of the Mahalanobis distance (standardized Euclidean distance), giving an intuitive way to scale the $f_{\mathrm{LL}}$ function to understand the model match score. Indeed, when the model is exact and $\mu_{k}=\tilde{\mu}_{k}$, we get $E\left[f_{\mathrm{LL}}^{\text {exact }}\left(\left\{m_{k}\right\} \mid \beta\right)\right]=0$. We note, however, that the function can take values below 0 as the variance for the exact case is $\operatorname{Var}\left[f_{\mathrm{LL}}^{\text {exact }}\left(\left\{m_{k}\right\} \mid \beta\right)\right]=1 /(2 K)$. Such values indicate the standard deviation expected by the model, $\widetilde{\sigma}_{k}$ is larger than the standard deviation observed experimentally, $\sigma_{k}$.

\section{Model analysis}

Both during and after the learning process, it is beneficial to interrogate the model to estimate its properties and their impact on the system behavior. As part of the $\mathbb{C}^{3}$ tool set, we perform sensitivity analysis for system parameters: sweeping a single parameter, e.g., qubit frequency, across the range of interest, while keeping other parameters at their current best value, evaluating the model match score at each point, as seen in the example [Fig. 4(a)]. The result is a $1 \mathrm{D}$ cut through the optimization landscape that may exhibit a well-defined minimum, multiple extrema indicating a difficult optimization, or even appear flat in the case when a parameter does not affect the behavior of the current experiment. This landscape depends on both the selected model and data it is compared to. Depending on the ruggedness of the sensitivity, one might choose to utilize a gradient-based algorithm from the start or to first perform a gradient-free exploratory search to avoid local minima. In the case of a flat sensitivity, there are two courses of action: if the parameter is of little interest for successive experiments, it may be removed or set 
to a convenient value within the flat range; otherwise, one needs to design an experiment producing additional data that are sensitive to the parameter. The physicists' knowledge of common experimental practices (e.g., Rabi, Ramsey, Hahn echo sequences) and intuition guides the decision for the experiment design. When suspecting correlations between parameters, cuts in single dimensions are not enough and higher-dimensional sweeps are necessary. After a successful learning process, the sensitivity analysis gives an estimate of the precision to which each parameter has been determined.

Furthermore, the simulation allows insight into the behavior of the system. Using well-established methods such as time-resolved state and process tomography, it is possible to identify coherent errors, such as leakage out of the computational subspace, over rotations, and the effects of noise. A Good Model also provides the basis for an error budget, as it contains the same limitations as the experiment it accurately predicts. The model can be used for extrapolation by idealizing certain aspects suspected as causes of infidelity (e.g., $T_{1}$ setting to infinity), and rederiving control pulses using a $C_{1}$ optimization. The respective gain in fidelity gives an estimate of the error that this aspect is responsible for, suggesting areas of improvement for future devices.

\section{DISCUSSION AND OUTLOOK}

In conclusion, we have described $\mathbb{C}^{3}$, an integrated methodology to improve quantum device performance that combines characterization, calibration, and control. We have detailed its approach and implementation, demonstrating, on a synthetic QPU device, the individual methods and how they are synthesized into a more integrated concept. Analyzing single-qubit calibration data we successfully extract an accurate model of the device, including realistic experimental considerations: line transfer functions, limitations of control electronics, readout error, and finite operating temperature. From this model we are able to derive a working high-fidelity two-qubit gate, without requiring any further calibration.

This approach represents a holistic theoretical take on the experimental workflow of a complex quantum computing experiment that takes into account interactions between different tasks of an experimental lab. The $\mathbb{C}^{3}$ methodology provides a path to achieve, starting from an incomplete understanding of the system, both high-fidelity pulses and an accurate model. It integrates the tasks of open-loop control (that would require an already accurate model) and of calibration (that would require an experiment-specific fine-tuning procedure). Most notably, it provides the tools to reflect on the experimental outcome and gate performance, improving the model description of the system and providing insight into its behavior. The $\mathbb{C}^{3}$ tool set is not a "black-box" experiment controller that replaces physicists or engineers - rather, it reduces tedious tasks allowing for interaction with the quantum device on a more structural level. Instead of simply producing high-fidelity operations, $\mathbb{C}^{3}$ provides meaningful output in the form of a Good Model of the system, and other insights such as an error budget and a sensitivity analysis. In this sense, $\mathbb{C}^{3}$ is not to be confused with any single optimal control or benchmarking technique, as it includes results from decades of research in these fields aimed at making controls that allow one to actually reach high fidelities efficiently $[8,50]$, unifying them into one framework.

We expect that the application of $\mathbb{C}^{3}$ will first benefit scalable implementations of quantum processors based on manufactured solid-state systems, such as superconducting and semiconducting qubits. There, the dependence of model parameters on fabrication means that many elements of the model are in fact uncertain. On the other hand, other scalable implementations of quantum computers contain such elements directly in their quantum description: ion trap gates involve degrees of freedom of the trap, impurity spins depend on their detailed position, etc. - thus we expect that $\mathbb{C}^{3}$ will also play a key role in those types of systems.

In the near future, we intend on extending the initial version of the $\mathbb{C}^{3}$ tool set to the generation of robust controls, automatic experiment design, multiparameter sensitivity analysis, active model learning, and more. The simulator will be enhanced to include non-Markovian noise, a detailed simulation of the readout process, echoes on control lines, and all other phenomena needed to produce a Good Model of real-world systems. The $\mathbb{C}^{3}$ tool set is currently being integrated with existing quantum computing software stacks, which would allow users to study custom pulse schedules $[94,95]$ and perform model learning based on data gathered from quantum computers, for example, with Qiskit Ignis [96]. Experimental application of $\mathbb{C}^{3}$ is ongoing (e.g., Ref. [32]).

It is our hope that $\mathbb{C}^{3}$ will not only provide insights into, and assist optimization of, current experiments, but also help guide the design of next-generation quantum devices, be it manually or by integration into automatic hardware design frameworks [76,77,97].

\section{ACKNOWLEDGMENTS}

This work is supported by the European Commission through the Marie Curie ETN QuSCo (Grant No. 765267) and the OpenSuperQ project (Grant No. 820363), by the Intelligence Advanced Research Projects Activity (IARPA) through the LogiQ (Grant No. W911NF-16-10114), and by the Germany Ministry of Science and Education (BMBF) through project VERTICONS (Grant No. 13N14872). 


\section{APPENDIX A: SURVEY OF PARAMETER-SPECIFIC CHARACTERIZATION}

The task of characterization of quantum devices in general has received extensive attention. It would be presumptuous of us to even attempt a complete survey; therefore, we limit ourselves to a very limited look at a subset of model-specific methods we have subjectively found informative to our own work.

The standard approach at addressing the lack of a Good Model, as defined above, is to perform a long list of model-specific characterization experiments, each designed to measure a different parameter of the model: measure parameters of the readout resonator using frequency sweeps; qubit frequency measurements and relaxation time $T_{1}$ require Rabi experiments [98] (and, with some extra effort, the higher levels can be extracted); Ramsey [99] and Hahn echo measurements [100] provide dephasing data (under the Markovian assumption, which is known to be an oversimplification $[101,102])$; measuring the control line response functions [103-106,106,107], the noise spectra [28,102,108,109], continuous drifts in system parameters [110-113], and discontinuous jumps in parameters such as $T_{1}[114,115]$; SPAM errors can be extracted from randomized benchmarking (e.g., Ref. [37]) or dedicated procedures, such as in Ref. [116]; qubit crosstalk can be measured by the method described in Refs. [117,118] and many more. Model-specific methods also exist for learning spin chain, lattice Hamiltonians, and other multiparticle systems with a predefined network topology under limited access [119-123].

\section{APPENDIX B: CHOICE OF GRADIENT-FREE ALGORITHMS}

Naturally, using an automated approach to solve the gradient-free optimization problem of calibration seems promising, and many experiments are currently using the Nelder-Mead algorithm [124,125] for their calibration tasks. As an example, in Fig. 9 we show an underlying parameter landscape of an optimization, obtained by simulating DRAG-corrected pulses. Calibration can be rather difficult as, depending on the starting position the optimizer, algorithms will have to overcome local minima and deal with intrinsic noise. It is further noteworthy that this landscape is rather unique to the used parametrization, the chosen goal function, and ultimately the properties of the physical system.

Research of gradient-free optimizers is a vast and active field, with hundreds of published black-box optimizers, including evolutionary strategies (ES), particle swarm optimization, differential evolution, random search, simultaneous perturbation stochastics approximation, the Nelder-Mead method, Bayesian optimization, and more. From a preliminary investigation, we recommend evolutionary strategies such as the CMA-ES algorithm, which is currently the default in $C_{2}$. This algorithm performed well in most cases, exhibited good robustness to noise, can handle local extrema, and requires relatively few evaluations. Similar conclusions have been reached independently in Ref. [126]. We make no claim as to the optimality of the optimizer chosen, and defer a more detailed discussion of the subject to future publications.

\section{APPENDIX C: OPEN-SOURCE IMPLEMENTATION}

The $\mathbb{C}^{3}$ tool set is implemented as an open-source project available online [127] under the Apache 2.0 license. The software is written in PYTHON to interface conveniently with common experiment controllers, and has already been used in tandem with PycQED [128], Labber
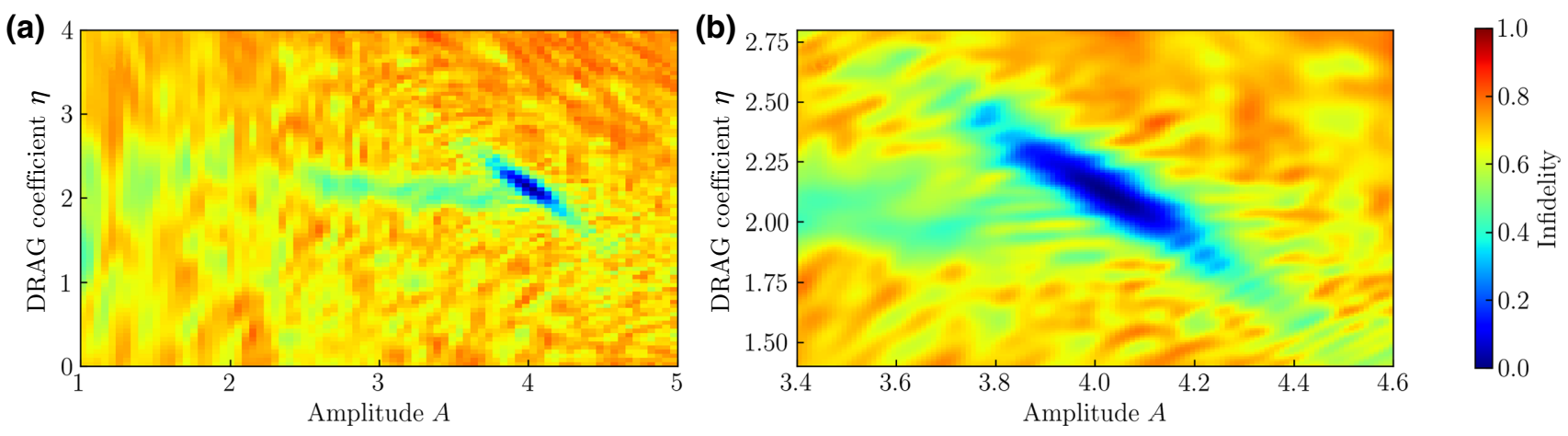

FIG. 9. A 2D cut through the landscape of a calibration goal function. The system chosen is a multilevel qubit, with the control sequence being a single length RB sequence. Control parameters $\alpha_{j}$ are the Gaussian pulse amplitude, DRAG coefficient, and frequency offset of a DRAG-corrected pulse. The RB sequence is chosen to implement the identity operation, ideally leaving the qubit in the ground state. The infidelity (seen as the color ranging from 0 to 1 ) is defined as the overlap of the final state with the system's ground state. (a) The landscape of the simulated system, as a cut through the plane of pulse amplitude $A$ and DRAG coefficient $\eta$, for a fixed RB sequence. Multiple local minima can be observed. (b) A higher-resolution plot of the same landscape. Further local minima can be observed in the neighborhood of the global minimum. 
[129], and LabView [130]. The interface can occur at various levels of abstraction, from sharing control parameters to sampled waveform values. A modular design allows for Hamiltonian or Lindbladian descriptions of common physical systems (fixed and flux-tunable qubits, resonators, different types of coupling), specification of a list of devices to model the signal chain of the experiment (local oscillator, AWG, mixers, distortions, and attenuations), different types of readout processing, and various fidelity functions. All components can be edited by the user or taken from reference libraries, accommodating to different needs. Configurations and data are stored as JSON files, and the full capabilities are accessible as command-line scripts, allowing for easy automation.

Numeric calculations are performed using TensorFlow [51]: the simulation of the dynamics and the pre- and postprocessing are formulated as a network, with welldefined inputs (e.g., control and model parameters) and outputs (goal function values), connected by many nodes, each performing a relatively simple operation (e.g., matrix exponentiation). TensorFlow enables the numerical computation of the Jacobian of a calculation - the gradient of each of the network outputs with respect to the network inputs (this capability is the evolution of what is known as a back-propagation learning process in neural networks [131]). This process of automatic differentiation facilitates the modular structure, as any new component inherits this property, removing the need to analytically derive its gradient. Furthermore, the TensorFlow simulator is scalable, allowing deployment on a variety of high-performance computing hardware.

We note prior efforts simulating quantum circuits that allow for automatic differentiation, e.g., Refs. [53,132], as well as large-scale simulations of quantum circuits, e.g., Refs. [133-135].

Each component of the control stack and model needs to conform to a general boilerplate that specifies what parameters it contains and how they are used. In this modular design, each class represents a component of the experiment that takes an input, applies some parameterdependent function to it, and returns a result. For example, an envelope function for pulses would have the following structure.

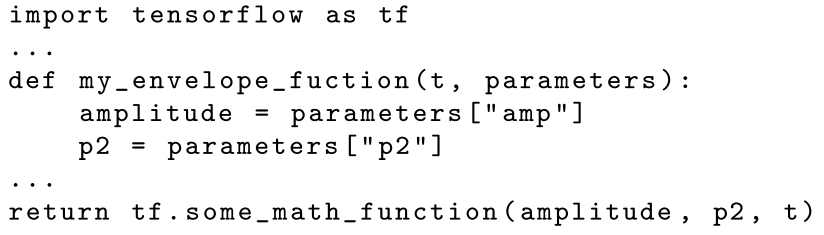

The only requirement to this code is that mathematical functions have to be taken from the TensorFlow package to allow for automatic differentiation. As an example of a control stack element, the finite rise time of an AWG is realized with the following code.

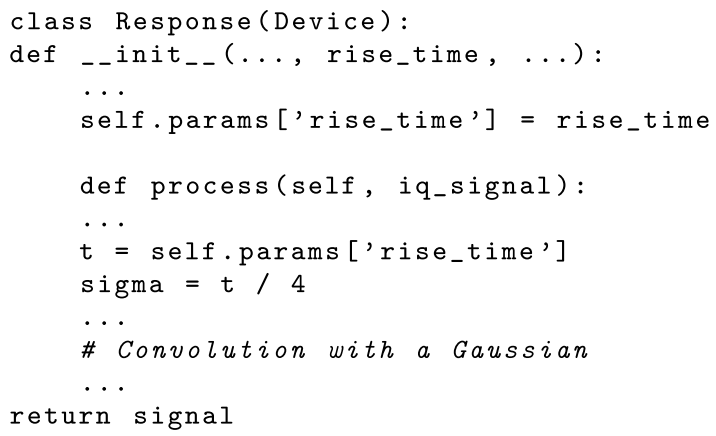

A signal processing chain is represented by putting the output of one control stack element into the next. In calculating figures of merit, the user can choose from a library of functions or supply their own. For example, the infidelity of a state transfer process from $\left|\psi_{0}\right\rangle$ to $\left|\psi_{\text {ideal }}\right\rangle$, implemented by the simulated propagator $U$ as follows.

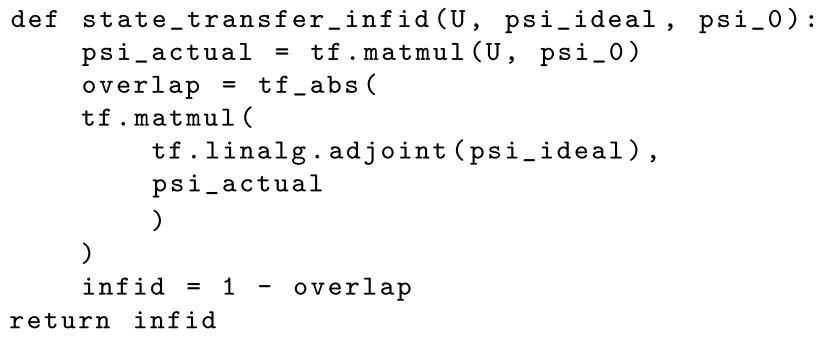

[1] P. Jurcevic, A. Javadi-Abhari, L. S. Bishop, I. Lauer, D. F. Bogorin, M. Brink, L. Capelluto, O. Günlük, T. Itoko, and N. Kanazawa et al., Demonstration of quantum volume 64 on a superconducting quantum computing system, Phys. Rev. Res. 2, 033447 (2020).

[2] F. Arute, K. Arya, R. Babbush, D. Bacon, J. C. Bardin, R. Barends, R. Biswas, S. Boixo, F. G. S. L. Brandao, and D. A. Buell et al., Quantum supremacy using a programmable superconducting processor, Nature 574, 505 (2019).

[3] A. W. Cross, L. S. Bishop, S. Sheldon, P. D. Nation, and J. M. Gambetta, Validating quantum computers using randomized model circuits, Phys. Rev. A 100, 032328 (2019).

[4] R. Barends et al., Superconducting quantum circuits at the surface code threshold for fault tolerance, Nature 508, 500 (2014).

[5] M. C. Collodo, J. Herrmann, N. Lacroix, C. K. Andersen, A. Remm, S. Lazar, J.-C. Besse, T. Walter, A. Wallraff, and C. Eichler, Implementation of conditional-phase gates based on tunable zz-interactions, arXiv:2005.08863 (2020).

[6] M. Ganzhorn, G. Salis, D. J. Egger, A. Fuhrer, M. Mergenthaler, C. Müller, P. Müller, S. Paredes, M. Pechal, M. Werninghaus, and S. Filipp, Benchmarking the noise sensitivity of different parametric two-qubit gates in a single superconducting quantum computing platform, Phys. Rev. Res. 2, 033447 (2020). 
[7] G. E. P. Box, Science and statistics, J. Am. Stat. Assoc. 71, 791 (1976).

[8] S. J. Glaser, U. Boscain, T. Calarco, C. P. Koch, W. Köckenberger, R. Kosloff, I. Kuprov, B. Luy, S. Schirmer, T. Schulte-Herbrüggen, D. Sugny, and F. K. Wilhelm, Training schrödinger's cat: Quantum optimal control, Eur. Phys. J. D 69, 279 (2015).

[9] S. Machnes, U. Sander, S. J. Glaser, P. de Fouquières, A. Gruslys, S. Schirmer, and T. Schulte-Herbrüggen, Comparing, optimizing, and benchmarking quantum-control algorithms in a unifying programming framework, Phys. Rev. A 84, 022305 (2011).

[10] S. Machnes, E. Assémat, D. Tannor, and F. K. Wilhelm, Tunable, Flexible, and Efficient Optimization of Control Pulses for Practical Qubits, Phys. Rev. Lett. 120, 150401 (2018).

[11] N. Khaneja, T. Reiss, C. Kehlet, T. Schulte-Herbrüggen, and S. J. Glaser, Optimal control of coupled spin dynamics: Design of nmr pulse sequences by gradient ascent algorithms, J. Magn. Reson. 172, 296 (2005).

[12] P. Doria, T. Calarco, and S. Montangero, Optimal Control Technique for Many-Body Quantum Dynamics, Phys. Rev. Lett. 106, 190501 (2011).

[13] R. Kosloff, S. A. Rice, P. Gaspard, S. Tersigni, and D. Tannor, Wavepacket dancing: Achieving chemical selectivity by shaping light pulses, Chem. Phys. 139, 201 (1989).

[14] F. Motzoi, J. M. Gambetta, P. Rebentrost, and F. K. Wilhelm, Simple Pulses for Elimination of Leakage in Weakly Nonlinear Qubits, Phys. Rev. Lett. 103, 110501 (2009).

[15] R. Schutjens, F. A. Dagga, D. J. Egger, and F. K. Wilhelm, Single-qubit gates in frequency-crowded transmon systems, Phys. Rev. A 88, 052330 (2013).

[16] U. Dorner, T. Calarco, P. Zoller, A. Browaeys, and P. Grangier, Quantum logic via optimal control in holographic dipole traps, J. Opt. B: Quantum Semiclassical Opt. 7, S341 (2005).

[17] D. J. Egger and F. K. Wilhelm, Optimized controlled-Z gates for two superconducting qubits coupled through a resonator, Supercond. Sci. Technol. 27, 014001 (2013).

[18] D. J. Egger and F. K. Wilhelm, Adaptive Hybrid Optimal Quantum Control for Imprecisely Characterized Systems, Phys. Rev. Lett. 112, 240503 (2014).

[19] J. Kelly et al., Optimal Quantum Control Using Randomized Benchmarking, Phys. Rev. Lett. 112, 240504 (2014).

[20] S. Sheldon, E. Magesan, J. M. Chow, and J. M. Gambetta, Procedure for systematically tuning up cross-talk in the cross-resonance gate, Phys. Rev. A 93, 060302(R) (2016).

[21] S. Sheldon, L. S. Bishop, E. Magesan, S. Filipp, J. M. Chow, and J. M. Gambetta, Characterizing errors on qubit operations via iterative randomized benchmarking, Phys. Rev. A 93, 012301 (2016).

[22] M. Gevers, Identification for control: From the early achievements to the revival of experiment design, Eur. J. Control 11, 335 (2005).

[23] C. R. Rojas, J. S. Welsh, G. C. Goodwin, and A. Feuer, Robust optimal experiment design for system identification, Automatica 43, 993 (2007).

[24] L. Ljung, Perspectives on system identification, Annu. Rev. Control 34, 1 (2010).
[25] X. Bombois, M. Gevers, R. Hildebrand, and G. Solari, Optimal experiment design for open and closed-loop system identification, Commun. Inf. Syst. 11, 197 (2011).

[26] Q.-M. Chen, X. Yang, C. Arenz, R.-B. Wu, X. Peng, I. Pelczer, and H. Rabitz, Combining the synergistic control capabilities of modelling and experiments: illustration of finding a minimum time quantum objective, arXiv:1812.05042 (2018).

[27] R.-B. Wu, B. Chu, D. H. Owens, and H. Rabitz, Datadriven gradient algorithm for high-precision quantum control, Phys. Rev. A 97, 042122 (2018).

[28] H. Ball, M. J. Biercuk, A. Carvalho, J. Chen, M. Hush, L. A. D. Castro, L. Li, P. J. Liebermann, H. J. Slatyer, C. Edmunds, V. Frey, C. Hempel, and A. Milne, Software tools for quantum control: Improving quantum computer performance through noise and error suppression, arXiv:2001.04060 [quant-ph] (2020).

[29] G. Lindblad, On the generators of quantum dynamical semigroups, Commun. Math. Phys. 48, 119 (1976).

[30] V. Gorini, A. Kossakowski, and E. C. G. Sudarshan, Completely positive dynamical semigroups of n-level systems, J. Math. Phys. 17, 821 (1976).

[31] B. Girod, R. Rabenstein, and A. Stenger, Signals and Systems (Wiley, Chichester, 2001).

[32] M. Werninghaus, D. J. Egger, F. Roy, S. Machnes, F. K. Wilhelm, and S. Filipp, Leakage reduction in fast superconducting qubit gates via optimal control, Npj Quantum Inf. 7, (2021).

[33] Z. Chen et al., Measuring and Suppressing Quantum State Leakage in a Superconducting Qubit, Phys. Rev. Lett. 116, 020501 (2016).

[34] E. Magesan, R. Blume-Kohout, and J. Emerson, Gate fidelity fluctuations and quantum process invariants, Phys. Rev. A 84, 012309 (2011).

[35] See Supplemental Material at http://link.aps.org/supple mental/10.1103/PhysRevApplied.15.034080 for additional sensitivity plots.

[36] J. Nocedal, Updating quasi-newton matrices with limited storage, Math. Comput. 35, 773 (1980).

[37] E. Knill, D. Leibfried, R. Reichle, J. Britton, R. B. Blakestad, J. D. Jost, C. Langer, R. Ozeri, S. Seidelin, and D. J. Wineland, Randomized benchmarking of quantum gates, Phys. Rev. A 77, 012307 (2008).

[38] J. M. Chow, J. M. Gambetta, L. Tornberg, J. Koch, L. S. Bishop, A. A. Houck, B. R. Johnson, L. Frunzio, S. M. Girvin, and R. J. Schoelkopf, Randomized Benchmarking and Process Tomography for Gate Errors in a Solid-State Qubit, Phys. Rev. Lett. 102, 090502 (2009).

[39] E. Magesan, J. M. Gambetta, and J. Emerson, Scalable and Robust Randomized Benchmarking of Quantum Processes, Phys. Rev. Lett. 106, 180504 (2011).

[40] E. Magesan, J. M. Gambetta, and J. Emerson, Characterizing quantum gates via randomized benchmarking, Phys. Rev. A 85, 042311 (2012).

[41] N. Hansen, S. D. Müller, and P. Koumoutsakos, Reducing the time complexity of the derandomized evolution strategy with covariance matrix adaptation (cma-es), Evol. Comput. 11, 1 (2003).

[42] M. Sinanovic, B.Sc. thesis, Saarland University (unpublished). 
[43] A. Blais, R.-S. Huang, A. Wallraff, S. M. Girvin, and R. J. Schoelkopf, Cavity quantum electrodynamics for superconducting electrical circuits: An architecture for quantum computation, Phys. Rev. A 69, 062320 (2004).

[44] J. J. Wallman and S. T. Flammia, Randomized benchmarking with confidence, New J. Phys. 16, 103032 (2014).

[45] C. Rigetti and M. Devoret, Fully microwave-tunable universal gates in superconducting qubits with linear couplings and fixed transition frequencies, Phys. Rev. B 81, 134507 (2010).

[46] J. M. Chow, A. D. Córcoles, J. M. Gambetta, C. Rigetti, B. R. Johnson, J. A. Smolin, J. R. Rozen, G. A. Keefe, M. B. Rothwell, M. B. Ketchen, and M. Steffen, Simple All-Microwave Entangling Gate for Fixed-Frequency Superconducting Qubits, Phys. Rev. Lett. 107, 080502 (2011).

[47] T. Alexander, N. Kanazawa, D. J. Egger, L. Capelluto, C. J. Wood, A. Javadi-Abhari, and D. McKay, Qiskit pulse: Programming quantum computers through the cloud with pulses, Quantum Sci. Technol. 5, 044006 (2020).

[48] A. D. Córcoles, J. M. Gambetta, J. M. Chow, J. A. Smolin, M. Ware, J. Strand, B. L. T. Plourde, and M. Steffen, Process verification of two-qubit quantum gates by randomized benchmarking, Phys. Rev. A 87, 030301(R) (2013).

[49] S. Kirchhoff, T. Keßler, P. J. Liebermann, E. Assémat, S. Machnes, F. Motzoi, and F. K. Wilhelm, Optimized crossresonance gate for coupled transmon systems, Phys. Rev. A 97, 042348 (2018).

[50] F. K. Wilhelm, S. Kirchhoff, S. Machnes, N. Wittler, and D. Sugny, An introduction into optimal control for quantum technologies, arXiv:2003.10132 (2020).

[51] M. Abadi, A. Agarwal, P. Barham, E. Brevdo, Z. Chen, C. Citro, G. S. Corrado, A. Davis, J. Dean, M. Devin, S. Ghemawat, I. Goodfellow, A. Harp, G. Irving, M. Isard, TensorFlow, Large-scale machine learning on heterogeneous systems, (2015), software available from tensorflow.org.

[52] G. Kedem, Automatic differentiation of computer programs, ACM Trans. Math. Software (TOMS) 6, 150 (1980).

[53] N. Leung, M. Abdelhafez, J. Koch, and D. Schuster, Speedup for quantum optimal control from automatic differentiation based on graphics processing units, Phys. Rev. A 95, 042318 (2017).

[54] D. C. McKay, C. J. Wood, S. Sheldon, J. M. Chow, and J. M. Gambetta, Efficient $z$ gates for quantum computing, Phys. Rev. A 96, 022330 (2017).

[55] D. A. Lidar, Lecture notes on the theory of open quantum systems, arXiv:1902.00967 (2019).

[56] S. V. Stehman, Selecting and interpreting measures of thematic classification accuracy, Remote Sens. Environ. 62, 77 (1997).

[57] A. P. Peirce, M. A. Dahleh, and H. Rabitz, Optimal control of quantum-mechanical systems: Existence, numerical approximation, and applications, Phys. Rev. A 37, 4950 (1988).

[58] D. M. Reich, M. Ndong, and C. P. Koch, Monotonically convergent optimization in quantum control using krotov's method, J. Chem. Phys. 136, 104103 (2012).
[59] M. H. Goerz, D. Basilewitsch, F. Gago-Encinas, M. G. Krauss, K. P. Horn, D. M. Reich, and C. P. Koch, Krotov: A python implementation of krotov's method for quantum optimal control, SciPost Phys. 7, 80 (2019).

[60] Z. Leng, P. Mundada, S. Ghadimi, and A. Houck, Robust and efficient algorithms for high-dimensional black-box quantum optimization, arXiv:1910.03591 (2019).

[61] J. Nocedal and S. Wright, Numerical Optimization (Springer Science \& Business Media, New York, 2006).

[62] G. Feng, F. H. Cho, H. Katiyar, J. Li, D. Lu, J. Baugh, and R. Laflamme, Gradient-based closed-loop quantum optimal control in a solid-state two-qubit system, Phys. Rev. A 98, 052341 (2018).

[63] J. Kelly, P. O’Malley, M. Neeley, H. Neven, and J. M. Martinis, Physical qubit calibration on a directed acyclic graph, arXiv:1803.03226 (2018).

[64] P. C. Mahalanobis, On the generalized distance in statistics Proc. Natn. Inst. Sci. 2, 49 (1936).

[65] S. G. Schirmer and D. K. L. Oi, Quantum system identification by bayesian analysis of noisy data: Beyond hamiltonian tomography, Laser Phys. 20, 1203 (2010).

[66] M. P. V. Stenberg, Y. R. Sanders, and F. K. Wilhelm, Efficient Estimation of Resonant Coupling between Quantum Systems, Phys. Rev. Lett. 113, 210404 (2014).

[67] M. P. V. Stenberg, O. Köhn, and F. K. Wilhelm, Characterization of decohering quantum systems: Machine learning approach, Phys. Rev. A 93, 012122 (2016).

[68] M. P. V. Stenberg and F. K. Wilhelm, Simultaneous model selection and parameter estimation: A superconducting qubit coupled to a bath of incoherent two-level systems, Phys. Rev. A 94, 052119 (2016).

[69] A. Sergeevich, A. Chandran, J. Combes, S. D. Bartlett, and H. M. Wiseman, Characterization of a qubit hamiltonian using adaptive measurements in a fixed basis, Phys. Rev. A 84, 052315 (2011).

[70] N. Wiebe, C. Granade, C. Ferrie, and D. G. Cory, Hamiltonian Learning and Certification Using Quantum Resources, Phys. Rev. Lett. 112, 190501 (2014).

[71] N. Wiebe, C. Granade, C. Ferrie, and D. Cory, Quantum hamiltonian learning using imperfect quantum resources, Phys. Rev. A 89, 042314 (2014).

[72] N. Wiebe, C. Granade, and D. G. Cory, Quantum bootstrapping via compressed quantum hamiltonian learning, New J. Phys. 17, 022005 (2015).

[73] J. Wang, S. Paesani, R. Santagati, S. Knauer, A. A. Gentile, N. Wiebe, M. Petruzzella, J. L. O’Brien, J. G. Rarity, and A. Laing et al., Experimental quantum hamiltonian learning, Nat. Phys. 13, 551 (2017).

[74] J. H. Cole, S. G. Schirmer, A. D. Greentree, C. J. Wellard, D. K. L. Oi, and L. C. L. Hollenberg, Identifying an experimental two-state hamiltonian to arbitrary accuracy, Phys. Rev. A 71, 062312 (2005).

[75] C. E. Granade, C. Ferrie, N. Wiebe, and D. G. Cory, Robust online hamiltonian learning, New J. Phys. 14, 103013 (2012).

[76] M. Krenn, M. Malik, R. Fickler, R. Lapkiewicz, and A. Zeilinger, Automated Search for new Quantum Experiments, Phys. Rev. Lett. 116, 090405 (2016).

[77] A. A. Melnikov, H. P. Nautrup, M. Krenn, V. Dunjko, M. Tiersch, A. Zeilinger, and H. J. Briegel, Active learning 
machine learns to create new quantum experiments, Proc. Natl. Acad. Sci. 115, 1221 (2018).

[78] M. Krenn, M. Erhard, and A. Zeilinger, Computerinspired quantum experiments, arXiv:2002.09970 [quantph] (2020).

[79] A. A. Gentile, B. Flynn, S. Knauer, N. Wiebe, S. Paesani, C. E. Granade, J. G. Rarity, R. Santagati, and A. Laing, Learning models of quantum systems from experiments, arXiv:2002.06169 (2020).

[80] T. J. Evans, R. Harper, and S. T. Flammia, Scalable bayesian hamiltonian learning, arXiv:1912.07636 (2019).

[81] J. F. Ralph, S. Maskell, and K. Jacobs, Multiparameter estimation along quantum trajectories with sequential monte carlo methods, Phys. Rev. A 96, 052306 (2017).

[82] R. L. Kosut and H. Rabitz, Identification of quantum systems, IFAC Proceedings Volumes 35, 397 (2002).

[83] S. G. Schirmer, A. Kolli, and D. K. L. Oi, Experimental hamiltonian identification for controlled two-level systems, Phys. Rev. A 69, 050306(R) (2004).

[84] D. K. L. Oi and S. G. Schirmer, Quantum system characterization with limited resources, Philos. Trans. Royal Society A: Math. Phys. Eng. Sci. 370, 5386 (2012).

[85] D. Burgarth and K. Yuasa, Quantum System Identification, Phys. Rev. Lett. 108, 080502 (2012).

[86] J. Zhang and M. Sarovar, Identification of open quantum systems from observable time traces, Phys. Rev. A 91, 052121 (2015).

[87] S.-Y. Hou, H. Li, and G.-L. Long, Experimental quantum hamiltonian identification from measurement time traces, Sci. Bull. 62, 863 (2017).

[88] A. Shabani, M. Mohseni, S. Lloyd, R. L. Kosut, and H. Rabitz, Estimation of many-body quantum hamiltonians via compressive sensing, Phys. Rev. A 84, 012107 (2011).

[89] K. Rudinger and R. Joynt, Compressed sensing for hamiltonian reconstruction, Phys. Rev. A 92, 052322 (2015).

[90] B. Lusch, J. N. Kutz, and S. L. Brunton, Deep learning for universal linear embeddings of nonlinear dynamics, Nat. Commun. 9, 1 (2018).

[91] E. Flurin, L. S. Martin, S. Hacohen-Gourgy, and I. Siddiqi, Using a Recurrent Neural Network to Reconstruct Quantum Dynamics of a Superconducting Qubit from Physical Observations, Phys. Rev. X 10, 011006 (2020).

[92] M. Dalgaard, F. Motzoi, J. J. Sørensen, and J. Sherson, Global optimization of quantum dynamics with alphazero deep exploration, npj Quantum Inf. 6, 1 (2020).

[93] S. Krastanov, S. Zhou, S. T. Flammia, and L. Jiang, Stochastic estimation of dynamical variables, Quantum Sci. Technol. 4, 035003 (2019).

[94] D. C. McKay, T. Alexander, L. Bello, M. J. Biercuk, L. Bishop, J. Chen, J. M. Chow, A. D. Córcoles, D. Egger, S. Filipp, J. Gomez, M. Hush, A. Javadi-Abhari, D. Moreda, P. Nation, B. Paulovicks, E. Winston, C. J. Wood, J. Wootton, and J. M. Gambetta, Qiskit backend specifications for openqasm and openpulse experiments, arXiv:1809.03452 (2018).

[95] T. Alexander, N. Kanazawa, D. J. Egger, L. Capelluto, C. J. Wood, A. Javadi-Abhari, and D. C. McKay, Qiskit pulse: programming quantum computers through the cloud with pulses, Quantum Sci. Technol. 5, 044006 (2020).
[96] G. Aleksandrowicz et al., Qiskit: An Open-source Framework for Quantum Computing (2019).

[97] B. P. MacLeod, F. G. L. Parlane, T. D. Morrissey, F. Häse, L. M. Roch, K. E. Dettelbach, R. Moreira, L. P. E. Yunker, M. B. Rooney, J. R. Deeth, V. Lai, G. J. Ng, H. Situ, R. H. Zhang, M. S. Elliott, T. H. Haley, D. J. Dvorak, A. Aspuru-Guzik, J. E. Hein, and C. P. Berlinguette, Selfdriving laboratory for accelerated discovery of thin-film materials, Sci. Adv. 6, eaaz8867 (2020).

[98] I. I. Rabi, Space quantization in a gyrating magnetic field, Phys. Rev. 51, 652 (1937).

[99] N. F. Ramsey, A molecular beam resonance method with separated oscillating fields, Phys. Rev. 78, 695 (1950).

[100] E. L. Hahn, Spin echoes, Phys. Rev. 80, 580 (1950).

[101] B. Pokharel, N. Anand, B. Fortman, and D. A. Lidar, Demonstration of Fidelity Improvement Using Dynamical Decoupling with Superconducting Qubits, Phys. Rev. Lett. 121, 220502 (2018).

[102] C. Ferrie, C. Granade, G. Paz-Silva, and H. M. Wiseman, Bayesian quantum noise spectroscopy, New J. Phys. 20, 123005 (2018).

[103] M. A. Rol, L. Ciorciaro, F. K. Malinowski, B. M. Tarasinski, R. E. Sagastizabal, C. C. Bultink, Y. Salathe, N. Haandbaek, J. Sedivy, and L. DiCarlo, Time-domain characterization and correction of on-chip distortion of control pulses in a quantum processor, Appl. Phys. Lett. 116, 054001 (2020).

[104] J. P. G. van Dijk, E. Kawakami, R. N. Schouten, M. Veldhorst, L. M. K. Vandersypen, M. Babaie, E. Charbon, and F. Sebastiano, Impact of Classical Control Electronics on Qubit Fidelity, Phys. Rev. Appl. 12, 044054 (2019).

[105] M. Jerger, A. Kulikov, Z. Vasselin, and A. Fedorov, In Situ Characterization of Qubit Control Lines: A Qubit as a Vector Network Analyzer, Phys. Rev. Lett. 123, 150501 (2019).

[106] I. N. Hincks, C. E. Granade, T. W. Borneman, and D. G. Cory, Controlling Quantum Devices with Nonlinear Hardware, Phys. Rev. Appl. 4, 024012 (2015).

[107] S. Gustavsson, O. Zwier, J. Bylander, F. Yan, F. Yoshihara, Y. Nakamura, T. P. Orlando, and W. D. Oliver, Improving Quantum Gate Fidelities by Using a Qubit to Measure Microwave Pulse Distortions, Phys. Rev. Lett. 110, 040502 (2013).

[108] R. S. Gupta, A. R. Milne, C. L. Edmunds, C. Hempel, and M. J. Biercuk, Adaptive scheduling of noise characterization in quantum computers, arXiv:1904.07225 (2019).

[109] R. Harper, S. T. Flammia, and J. J. Wallman, Efficient learning of quantum noise, arXiv:1907.13022 (2019).

[110] J. Kelly, R. Barends, A. G. Fowler, A. Megrant, E. Jeffrey, T. C. White, D. Sank, J. Y. Mutus, B. Campbell, and Y. Chen et al., Scalable in situ qubit calibration during repetitive error detection, Phys. Rev. A 94, 032321 (2016).

[111] L. Casparis, T. W. Larsen, M. S. Olsen, F. Kuemmeth, P. Krogstrup, J. Nygård, K. D. Petersson, and C. M. Marcus, Gatemon Benchmarking and Two-Qubit Operations, Phys. Rev. Lett. 116, 150505 (2016).

[112] C. Miquel, J. P. Paz, and W. H. Zurek, Quantum Computation with Phase Drift Errors, Phys. Rev. Lett. 78, 3971 (1997). 
[113] R. C. Bialczak, R. McDermott, M. Ansmann, M. Hofheinz, N. Katz, E. Lucero, M. Neeley, A. D. O'Connell, H. Wang, A. N. Cleland, and J. M. Martinis, 1/f Flux Noise in Josephson Phase Qubits, Phys. Rev. Lett. 99, 187006 (2007).

[114] J. J. Burnett, A. Bengtsson, M. Scigliuzzo, D. Niepce, M. Kudra, P. Delsing, and J. Bylander, Decoherence benchmarking of superconducting qubits, Npj Quantum Inf. 5, 1 (2019).

[115] M. Y. Niu et al., Learning non-Markovian quantum noise from moiré-enhanced swap spectroscopy with deep evolutionary algorithm, arXiv:1912.04368 (2019).

[116] A. Zhang, J. Xie, H. Xu, K. Zheng, H. Zhang, Y.T. Poon, V. Vedral, and L. Zhang, Experimental SelfCharacterization of Quantum Measurements, Phys. Rev. Lett. 124, 040402 (2020).

[117] P. Mundada, G. Zhang, T. Hazard, and A. Houck, Suppression of Qubit Crosstalk in a Tunable Coupling Superconducting Circuit, Phys. Rev. Appl. 12, 054023 (2019).

[118] P. Murali, D. C. McKay, M. Martonosi, and A. Javadi-Abhari, Software mitigation of crosstalk on noisy intermediate-scale quantum computers, arXiv:2001.02826 (2020).

[119] D. Burgarth, K. Maruyama, and F. Nori, Coupling strength estimation for spin chains despite restricted access, Phys. Rev. A 79, 020305(R) (2009).

[120] C. Di Franco, M. Paternostro, and M. S. Kim, Hamiltonian Tomography in an Access-Limited Setting Without State Initialization, Phys. Rev. Lett. 102, 187203 (2009).

[121] L. E. De Clercq, R. Oswald, C. Flühmann, B. Keitch, D. Kienzler, H.-Y. Lo, M. Marinelli, D. Nadlinger, V. Negnevitsky, and J. P. Home, Estimation of a general timedependent hamiltonian for a single qubit, Nat. Commun. 7, 1 (2016).

[122] A. Sone and P. Cappellaro, Hamiltonian identifiability assisted by a single-probe measurement, Phys. Rev. A 95, 022335 (2017).
[123] P. Yang, M. Yu, R. Betzholz, C. Arenz, and J. Cai, Complete Quantum-State Tomography with a Local Random Field, Phys. Rev. Lett. 124, 010405 (2020).

[124] J. A. Nelder and R. Mead, A simplex method for function minimization, Comput. J. 7, 308 (1965).

[125] L. Han and M. Neumann, Effect of dimensionality on the nelder-mead simplex method, Optim. Method. Softw. 21, 1 (2006).

[126] J. Rapin, M. Gallagher, P. Kerschke, M. Preuss, and O. Teytaud, in Proceedings of the Genetic and Evolutionary Computation Conference Companion, GECCO '19 (Association for Computing Machinery, New York, NY, USA, 2019), p. 1888.

[127] https://q-optimize.org.

[128] M. A. Rol, C. Dickel, S. Asaad, N. K. Langford, C. C. Bultink, R. Sagastizabal, N. K. Langford, G. de Lange, X. Fu, S. R. de Jong, F. Luthi, W. Vlothuizen, and M. S. Moreira, Pycqed_py3 (2019).

[129] Labber.org, Labber - software for instrument control and lab automation (2020).

[130] C. J. Kalkman, Labview: A software system for data acquisition, data analysis, and instrument control, J. Clin. Monit. 11, 51 (1995).

[131] D. E. Rumelhart, G. E. Hinton, and R. J. Williams, Learning representations by back-propagating errors, Nature 323, 533 (1986).

[132] M. Abdelhafez, D. I. Schuster, and J. Koch, Gradientbased optimal control of open quantum systems using quantum trajectories and automatic differentiation, Phys. Rev. A 99, 052327 (2019).

[133] D. Willsch, M. Nocon, F. Jin, H. De Raedt, and K. Michielsen, Gate-error analysis in simulations of quantum computers with transmon qubits, Phys. Rev. A 96, 062302 (2017).

[134] D. Willsch, M. Willsch, F. Jin, H. De Raedt, and K. Michielsen, Testing quantum fault tolerance on small systems, Phys. Rev. A 98, 052348 (2018).

[135] D. Willsch, Supercomputer simulations of transmon quantum computers, arXiv:2008.13490 (2020). 